\title{
INÉGALITÉS VARIATIONNELLES NON CONVEXES
}

\author{
Messaoud Bounkhel $^{1}$ et Djalel Bounkhel ${ }^{2}$
}

\begin{abstract}
Résumé. Dans cet article nous proposons différents algorithmes pour résoudre une nouvelle classe de problèmes variationels non convexes. Cette classe généralise plusieurs types d'inégalités variationnelles (Cho et al. (2000), Noor (1992), Zeng (1998), Stampacchia (1964)) du cas convexe au cas non convexe. La sensibilité de cette classe de problèmes variationnels non convexes a été aussi étudiée.
\end{abstract}

\begin{abstract}
In this paper we propose several algorithms of the projection type to solve a new class of nonconvex variational problems. This class generalizes many types of variational inequalities (Cho et al. (2000), Noor (1992), Zeng (1998), Stampacchia (1964)) from the convex case to the nonconvex case. The sensitivity of this class of nonconvex variational problems is also studied.
\end{abstract}

Classification Mathématique. 58E35, 49J40, 49J53, 49J52.

Received February 13, 2004. Revised November 30, 2004.

\section{Notations ET PRÉLIMinaires}

Dans tout ce qui suit $H$ est un espace de Hilbert réel muni de produit scalaire $<.$, . $>$, et de la norme associée $\|\cdot\|$. B dénote la boule unité fermée de $H$ centrée à l'origine. Si $S$ est un sous-ensemble fermé de $H$, $d(S, \cdot)$ (respectivement $\chi_{S}($.$) et \sigma(S,$.$) ) représente la fonction distance (respectivement la fonction indicatrice$ et la fonction support) associée à $S$. La projection sur $S$ est définie par :

$$
\operatorname{Proj}_{S}(u):=\{y \in S:\|u-y\|=d(S, u)\}, \text { pour tout } u \in H
$$

Le cône normal à un ensemble convexe fermé $S$ en $x \in S$ au sens d'analyse convexe est défini par :

$$
N^{\text {Conv }} \cdot(S ; x)=\{y \in H:<y, z-x>\leq 0, \quad \forall z \in S\}
$$

Remarquons que l'on a toujours $0 \in N^{C o n v} \cdot(S ; x)$ et $N^{C o n v} \cdot(\{x\} ; x)=H$ et que pour tout $x \in \operatorname{int}(S)$ on a $N^{\text {Conv. }}(S ; x)=\{0\}$.

Mots Clés. Ensembles uniformément réguliers, problèmes variationnels non convexes.

${ }^{1}$ King Saud University, College of Science, Department of Mathematics, PO Box 2455, Riyadh 11451, Saudi Arabia; bounkhel@ksu.edu . sa

2 University of Jijel, Department of Mathematics, BP 98, Ouled Aissa, Jijel, Algeria; bounkheldjalel@yahoo.fr

(c) EDP Sciences, SMAI 2005 
Soient $A$ et $B$ deux sous-ensembles fermés non vides de $H$. On appelle distance de Hausdorff entre $A$ et $B$ la fonction : $\mathcal{H}: H \times H \longrightarrow[0,+\infty]$ définie par :

$$
\mathcal{H}(A, B)=\max \left\{\sup _{a \in A} d(B, a), \sup _{b \in B} d(A, b)\right\} .
$$

Définition 1.1. Soit $\Phi: X \rightrightarrows Y$ une multifonction définie entre deux espaces topologiques $X$ et $Y$.

- On dit que $\Phi$ est semi-continue supérieurement (s.c.s.) en $x \in \operatorname{dom}(\Phi):=\{u \in X: \Phi(u) \neq \emptyset\}$ si pour tout ouvert $O$ contenant $\Phi(x)$ il existe un voisinage $V$ de $x$, tel que $\Phi(V) \subset O$.

- Si $\Phi: H \rightrightarrows H$ est à valeurs fermées convexes faiblement compactes, on dira que $\Phi$ est s.c.s. pour la topologie faible $\sigma(H, H)$ (ou bien qu'elle est scalairement s.c.s.) si pour tout $y$ dans $H$ la fonction support associée à $\Phi$ i.e., $\sigma(\Phi(\cdot), y)$ est s.c.s. (voir [4] Th. II. 20).

- Si $\Phi$ : $H \rightrightarrows H$ est à valeurs fermées non vides, on dira que $\Phi$ est lipschitzienne au sens de Hausdorff s'il existe une constante $\lambda>0$ tel que pour tout $x, y$ dans $H$ on a

$$
\mathcal{H}(\Phi(x), \Phi(y)) \leq \lambda\|x-y\|
$$

Définition 1.2. Le sous différentiel proximal d'une fonction semi-continue inférieure $f$ au point $x$, noté $\partial^{P} f(x)$, est l'ensemble de tous les $\xi \in H$, pour lesquels il existe $\sigma, \delta>0$ tels que pour tout $x^{\prime} \in x+\delta B$, on a :

$$
<\xi, x^{\prime}-x>\leq f\left(x^{\prime}\right)-f(x)+\sigma\left\|x^{\prime}-x\right\|^{2} .
$$

Définition 1.3. Soient $S$ un sous-ensemble fermé non vide de $H$ et $x \in S$. On définit le cône normal proximal de $S$ en $x$ par : $N^{P}(S, x)=\partial^{P} \chi_{S}(x)$, ce qui donne,

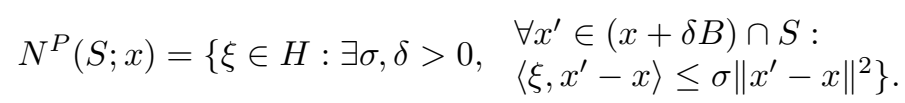

Le cône normal proximal est aussi donné, d'une manière équivalente, par :

$$
\begin{aligned}
N^{P}(S ; x) & =\left\{\xi \in H: \exists \alpha>0: x \in \operatorname{Proj}_{S}(x+\alpha \xi)\right\} \\
& =\{\xi \in H: \exists \alpha>0: d(S, x+\alpha \xi)=\alpha\|\xi\|\} .
\end{aligned}
$$

Observons que l'inégalite (1.6) dans la définition du cône normal proximal est verifiée localement et autour du point $x$. Dans la proposition 1.1.5 dans [8], les auteurs ont prouvé une caractérisation du cône normal proximal $N^{P}(S ; x)$ avec l'inégalité (1.6) et verifiée globalement pour tout $x^{\prime} \in S$. Nous la citons dans la proposition suivante.

Proposition 1.4. Soient $S$ un sous-ensemble fermé non vide de $H$ et $x \in S$. Alors

$$
N^{P}(S ; x)=\left\{\xi \in H: \exists \sigma>0:\left\langle\xi, x^{\prime}-x\right\rangle \leq \sigma\left\|x^{\prime}-x\right\|^{2}, \forall x^{\prime} \in S\right\}
$$

La relation entre le cône normal proximal et le sous différentiel proximal de la fonction distance est donnée par la proposition suivante due à [2].

Proposition 1.5. Soit $X$ un espace vectoriel normé, $S$ un sous-ensemble non vide fermé de $X$ et $x \in S$. Alors

$$
\partial^{P} d(S, x)=N^{P}(S ; x) \cap \mathbb{B}^{*}
$$

où $\mathbb{B}^{*}$ est la boule unité fermée de $X^{*}$. 
Remarque 1.6. D'autres concepts de sous différentiel (respectivement de cône normal) pour les fonctions (respectivement pour des ensembles non convexes) nondifférentiables ont été introduits et étudiés par plusieurs auteurs, nous citons entre autres le sous différentiel de Clarke et le cône normal de Clarke, le sous différentiel de Fréchet et le cône normal de Fréchet, le sous différentiel de Mordukhovich et le cône normal de Mordukhovich, etc. On réfère à $[2,3,6-8,11,12]$ pour les définitions de ces sous différentiels et de ces cônes normaux. Dans ce travail nous allons utiliser le sous différentiel proximal et le cône normal proximal car d'une part la classe d'ensembles avec laquelle nous allons travailler a été caractérisée en terme du sous différentiel proximal et du cône normal proximal et aussi parce que (voir la proposition suivante) tous les sous différentiels (resp. les cônes normaux) contenus dans le sous différentiel (resp. le cône normal) Clarke coïncident. Cette classe d'ensembles est appelée la classe des ensembles uniformément prox-réguliers.

Définition 1.7. Étant donné un $r \in] 0,+\infty]$, un sous-ensemble $S$ est uniformément $r$-prox-régulier si et seulement si pour tout $y \in S$ et tout $\xi \in N^{P}(S ; y),(\xi \neq 0)$ on a

$$
\left\langle\frac{\xi}{\|\xi\|}, x-y\right\rangle=\frac{1}{2 r}\|x-y\|^{2}, \text { pour tout } x \in S
$$

Par convention $\frac{1}{r}=0$ pour $r=+\infty$ (dans ce cas, l'uniforme $r$-prox-régularité est équivalente à la convexité de $S$ ). Quelques propriétés importantes de ces ensembles sont résumées dans la proposition suivante (voir les démonstrations dans $[3,7,11])$.

Proposition 1.8. Soit $r \in] 0,+\infty]$ et $S$ un sous-ensemble non vide fermé uniformément $r$-prox-régulier de $H$. Alors on $a$ :

(1) Pour tout $x \in H$ avec $d(S, x)<r$, la projection $\operatorname{Proj}_{S}(x)$ existe et elle est unique.

(2) Le sous différentiel proximal de $d(S, \cdot)$ coïncide avec tous les sous différentiels contenus dans le sous différentiel de Clarke en tout point $x \in H$ satisfaisant $d(S, x)<r$.

(3) Pour tout $\left.r^{\prime} \in\right] 0, r$ [, l'opérateur projection est lipschitzienne sur l'ensemble des points $x \in H$ satisfaisant $d(S, x)<r^{\prime}$, avec une constante de Lipschitz

$$
\zeta=\frac{r}{r-r^{\prime}}
$$

Dans toute la suite nous allons noter par $\partial d(S, x)$ le sous différentiel de la fonction distance associé à un sous-ensemble non vide fermé uniformément $r$-prox-régulier de $H$ (sans préciser le nom car ils sont tous égaux).

Comme conséquence de (2) dans la proposition précédente, pour les ensembles uniformément $r$-prox réguliers, le cône normal proximal à $S$ cö̈ncide avec tous les cônes normaux contenus dans le cône normal de Clarke en tout point $x \in S$, c.-à-d., $N^{P}(S ; x)=N^{C}(S ; x)$.

\section{Exemple 1.9.}

(1) Tous les ensembles convexes sont des ensembles uniformément $r$-prox-réguliers avec $r=+\infty$. Dans ce cas tous les cônes normaux coincident avec $N^{C o n v} \cdot(S ; x)$.

(2) L'union de deux intervalles disjoints $[a, b]$ et $[c, d]$ n'est pas convexe mais elle est uniformément $r$-prox-regulière avec $r=\frac{c-b}{2}$. On peut montrer que l'union finie des intervalles disjoints est toujours uniformément prox-régulière.

(3) L'union de deux ensembles convexes disjoints n'est pas convexe mais elle est uniformément $r$-proxregulière avec $r$ et égale à la moitié de la distance entre les deux ensembles.

Énonçons à présent une caractérisation très utile des ensembles uniformément $r$-prox-réguliers obtenue dans [3].

Proposition 1.10. Soient $r \in] 0,+\infty]$ et $S$ un sous-ensemble non vide fermé uniformément r-prox-régulier de $H$. Alors pour tout $x \in S, \xi \in \partial^{P} d(S, x)$ on $a$ :

$$
\left\langle\xi, x^{\prime}-x\right\rangle \leq \frac{2}{r}\left\|x^{\prime}-x\right\|^{2}+d\left(S, x^{\prime}\right), \text { pour tout } x^{\prime} \in H, \text { avec } d\left(S, x^{\prime}\right) \leq r .
$$


Cette proposition est utilisée pour montrer une propriété de fermeture du sous différentiel de la fonction distance associé à une multifonction à valeurs non vide fermées uniformément $r$-prox-régulières [3]. Cette propriété de fermeture à été prouvée dans [3]. Nous allons présenter sa démonstration ici pour l'intérêt des lecteurs. Tout d'abord nous rappelons la définition d'une multifonction lipschitzienne : une multifonction $K: H \rightrightarrows H$ est dite lipschitzienne s'il existe une constante $\kappa>0$ satisfaisant

$$
\left|d(K(x), y)-d\left(K\left(x^{\prime}\right), y^{\prime}\right)\right| \leq\left\|y^{\prime}-y\right\|+\kappa\left\|x^{\prime}-x\right\|
$$

pour tout $x, y, x^{\prime}, y^{\prime} \in H$. On dit aussi que $K$ est lipschitzienne avec une constante $\kappa$. Il est facile de vérifier que cette notion de Lipschitz est plus faible que la notion de Lipschitz au sens de Hausdorff donnée au début de ce section.

Proposition 1.11. Soient $r \in] 0,+\infty]$ et $K: H \rightrightarrows H$ une multifonction lipschitzienne telle que $K(y)$ soit uniformément $r$-prox-régulier pour tout $y \in H$. Pour toutes suites $x_{n}, y_{n}, \xi_{n}$ dans $H$ telles que $x_{n} \in K\left(y_{n}\right)$, $y_{n} \in H, \xi_{n} \in N^{P}\left(K\left(y_{n}\right) ; x_{n}\right)$, avec $x_{n} \rightarrow x_{0}, y_{n} \rightarrow y_{0}$, et $\xi_{n} \rightarrow \xi_{0}$, on a :

$$
\xi_{0} \in N^{P}\left(K\left(y_{0}\right) ; x_{0}\right) .
$$

Preuve. Soient $x_{n} \rightarrow x_{0}, y_{n} \rightarrow y_{0}$ et $\xi_{n} \rightarrow \xi_{0}$ avec $x_{n} \in K\left(y_{n}\right)$ and $\xi_{n} \in N^{P}\left(K\left(y_{n}\right) ; x_{n}\right)$. Si $\xi_{0}=0$, alors (1.13) est verifiée. Supposons maintenant que $\xi_{0} \neq 0$ (donc $\xi_{n} \neq 0$, pour $n$ suffisamment grand). Observons que $x_{0} \in K\left(y_{0}\right)$ parce que $K$ est lipschitzienne. La convergence de $x_{n}$ vers $x_{0}$ nous donne pour $n$ suffisamment grand

$$
x_{n} \in x_{0}+\frac{r}{2} \mathbb{B} .
$$

Par conséquent, l'uniforme prox-regularité des ensembles $K\left(y_{n}\right)$ et la proposition 1.10 nous donnent

$$
\left\langle\frac{\xi_{n}}{\left\|\xi_{n}\right\|}, z-x_{n}\right\rangle \leq \frac{2}{r}\left\|z-x_{n}\right\|^{2}+d\left(K\left(y_{n}\right), z\right), \forall z \in H, \text { avec } d\left(K\left(y_{n}\right), z\right)<r
$$

Cette inegalité est vraie pour tout $n$ suffisamment grand et pour tout $z \in x_{0}+\delta \mathbb{B}$ avec $0<\delta<\frac{r}{2}$. En effet pour tout $z \in x_{0}+\delta \mathbb{B}$ on a

$$
d\left(K\left(y_{n}\right), z\right) \leq\left\|z-x_{0}\right\|+\left\|x_{0}-x_{n}\right\| \leq \delta+\frac{r}{2}<r .
$$

Par conséquent, la continuité de la fonction distance $d(K(\cdot), \cdot)$ par rapport aux deux variables (car $K$ est Lipschitzienne) et la précédente inégalité donnent après passage à la limite quand $n \rightarrow+\infty$

$$
\left\langle\frac{\xi_{0}}{\left\|\xi_{0}\right\|}, z-x_{0}\right\rangle \leq \frac{2}{r}\left\|z-x_{0}\right\|^{2}+d\left(K\left(y_{0}\right), z\right), \text { pour tout } z \in x_{0}+\delta \mathbb{B} .
$$

D'où,

$$
\left\langle\frac{\xi_{0}}{\left\|\xi_{0}\right\|}, z-x_{0}\right\rangle \leq \frac{2}{r}\left\|z-x_{0}\right\|^{2}, \text { pour tout } z \in x_{0}+\delta \mathbb{B} \cap K\left(y_{0}\right) .
$$

Ceci assure, d'après la définition du cône normal proximal que $\frac{\xi_{0}}{\left\|\xi_{0}\right\|} \in N^{P}\left(K\left(y_{0}\right) ; x_{0}\right)$, ce qui donne $\xi_{0} \in$ $N^{P}\left(K\left(y_{0}\right) ; x_{0}\right)$. La preuve donc est terminée.

\section{Remarque 1.12.}

- Comme conséquence directe de ce résultat et de la proposition 1.5, on a la semi-continuité supérieure de la multifonction $(y, x) \rightrightarrows \partial^{P} d(K(y), x)$ de $H \times H$ vers $H \times H$, ce qui donne (voir par exemple Prop. 1.4.1 et Th. 1.4.2 dans 4) la semi-continuité supérieure de la fonction $(y, x) \mapsto \sigma\left(\partial^{P} d(K(y), x), p\right)$ pour tout $p \in H$. 
- En suivant la terminologie utilisée dans [4] ainsi que leurs références, on peut dire que la multifonction $K: H \rightrightarrows H$ est scalairement semi-continue supérieure sur $H$ si et seulement si pour tout $p \in H$, les fonctions supports $\sigma(K(\cdot), p)$ sont semi-continues supérieurement sur $H$.

- Dans le cas où $H=R^{n}$, et $K$ est à valeurs convexes, la semi-continuité supérieure de $K$ est équivalente à sa semi-continuité supérieure scalaire. Pour plus de détails, on réfère le lecteur à [4].

\section{Problèmes VARIATIONNELS NON CONVEXES}

Considérons un ensemble fermé non vide $C$, des multifonctions $F, G, S: H \rightrightarrows H$ et des applications $T, g$ : $H \rightarrow H$, et $h: H \times H \rightarrow H$, et soit $f \in H$. Dans [5], les auteurs ont introduit et étudié l'inégalité variationnelle suivante :

$$
\begin{aligned}
& \text { trouver } u^{*} \in H, x^{*} \in F\left(u^{*}\right), y^{*} \in G\left(u^{*}\right), w^{*} \in S\left(u^{*}\right) \text { et } \rho>0 \text { tels que : } \\
& \qquad g\left(u^{*}\right) \in C \text { et }\left\{\begin{array}{l}
\left\langle h\left(y^{*}, g\left(w^{*}\right)\right)-T\left(x^{*}\right)-f, v-g\left(u^{*}\right)\right\rangle \leq 0 \\
\forall v \in C .
\end{array}\right.
\end{aligned}
$$

Ce problème est appelé Inégalité Variationnelle Implicite et Nonlinéaire (IVIN). Il a été motivé par des applications en élasto-plasticité (voir $[5,10,13])$. Dans [5], les auteurs ont considéré et montré la convergence d'un algorithme vers une solution de (IVIN) sous certaines conditions et hypothèses sur $g, h, T, S, G, F$ et avec la convexité de l'ensemble $C$. Toutes les démonstrations et les analyses faites dans [5] sont fortement basées sur la convexité de $C$ et plus précisément sur les excellentes propriétés de la projection sur les ensembles convexes. D'où la généralisation directe du problème (IVIN) du cas convexe au cas non convexe, (i.e., considérer $C$ comme non convexe), n'est pas la bonne. Pour ceci nous allons suivre l'idée de [1] qui consiste à reformuler (IVIN) et la réécrire en terme du cône normal que l'on notera (PVIN), puis nous utiliserons des idées et des techniques de l'analyse non lisse et en particulier les propriétés des cônes normaux, pour proposer et analyser des algorithmes pour (PVIN).

Proposition 2.1. Supposons que $C$ est un sous-ensemble convexe fermé non vide de H, alors (IVIN) est équivalent au problème variationnel suivant :

$$
\begin{aligned}
& \text { trouver } u^{*} \in H \text { et } \rho>0 \text { tels que: } \\
& \qquad 0 \in\left\{h\left(G\left(u^{*}\right), g\left(S\left(u^{*}\right)\right)\right)-\rho\left[T\left(F\left(u^{*}\right)\right)-f\right]-N\left(C, g\left(u^{*}\right)\right)\right\} .
\end{aligned}
$$

Preuve. Elle suit directement de la définition du cône normal au sens d'analyse convexe.

Soit maintenant $C$ un sous-ensemble fermé non vide de $H$ (non nécessairement convexe). On a vu dans la section 1 qu'on peut considérer plusieurs concepts du cône normal à $C$ (par exemple : $N^{P}(C ; \cdot), N^{F}(C ; \cdot)$, $\left.N^{C}(C ; \cdot), \ldots\right)$. À chaque concept on peut associer un problème (PVIN).

Dans la proposition suivante nous écrivons le problème (PVIN) associé au cône normal proximal, que l'on note (PVINProx) sous forme d'inégalité variationnelle.

Proposition 2.2. Soit $C$ un sous-ensemble fermé non vide de H. Alors (PVINProx) est équivalent à l'inégalité variationnelle suivante (IVINProx) que l'on va appeler : Inégalité Variationnelle Implicite Nonlinéaire et Proximale :

$$
\begin{aligned}
& \text { trouver } u^{*} \in H, x^{*} \in F\left(u^{*}\right), y^{*} \in G\left(u^{*}\right), w^{*} \in S\left(u^{*}\right) \text { et } \sigma, \rho>0 \text { tels que: } \\
& \qquad g\left(u^{*}\right) \in C \text { et }\left\{\begin{array}{l}
\left\langle h\left(y^{*}, g\left(w^{*}\right)\right)-\rho\left[T\left(x^{*}\right)-f\right], v-g\left(u^{*}\right)\right\rangle \leq \sigma\left\|v-g\left(u^{*}\right)\right\|^{2} \\
\forall v \in C .
\end{array}\right.
\end{aligned}
$$

Preuve. Elle suit directement de la caractérisation du cône normal proximal prouvé dans la proposition 1.4. 


\section{Remarque 2.3.}

(1) D'après l'exemple 1.9-(1), la proposition 2.2 se réduit à la proposition 2.1, lorsque $C$ est un convexe fermé.

(2) En variant les concepts du cône normal, on peut obtenir plusieurs problèmes (PVIN) qui seront équivalents à différentes formes d'inégalités variationnelles.

(3) Dans ce travail nous sommes intéressés par la nouvelle classe d'ensembles non convexes appelée la classe des ensembles uniformément prox-réguliers, que l'on avait rappelée dans la section 1. L'une des importantes propriétés de cette classe est la coïncidence de tous les cônes normaux contenus dans le cône normal de Clarke, c.-à-d., $N^{P}(C,)=.N^{F}(C,)=.\ldots=N^{C}(C,$.$) . D'où la coïncidence de tous les$ problèmes (PVIN) associés aux ensembles $C$ uniformément prox-réguliers. Dans toute la suite on notera par $N(C,):.=N^{P}(C,)=.\ldots=N^{C}(C,$.$) pour tout C \in \operatorname{upr}(H)$ (i.e. les sous-ensembles uniformément prox-réguliers de $H$ ).

La proposition suivante établit une reformulation de (PVIN) associée à un ensemble $C \in \operatorname{upr}(H)$, sous forme d'inégalité variationnelle.

Proposition 2.4. Soit $C \in \operatorname{upr}(H)$ avec une constante $r \in] 0,+\infty]$. Alors le problème (PVIN) est équivalent à l'inégalité variationnelle suivante :

$$
\begin{aligned}
& \text { trouver } u^{*} \in H, x^{*} \in F\left(u^{*}\right), y^{*} \in G\left(u^{*}\right), w^{*} \in S\left(u^{*}\right) \text { et } \rho>0 \text { tels que : } \\
& g\left(u^{*}\right) \in C \text { et }\left\{\begin{array}{l}
\left\langle l, v-g\left(u^{*}\right)\right\rangle \leq \frac{1}{2 r}\|l\|\left\|v-g\left(u^{*}\right)\right\|^{2}, \quad \forall v \in C \\
\text { avec } l=h\left(y^{*}, g\left(w^{*}\right)\right)-\rho\left[T\left(x^{*}\right)-f\right] .
\end{array} \quad \text { (IVIN }\right)_{\text {upr }}
\end{aligned}
$$

Preuve. ( $\Leftarrow$ ?) Soient $u^{*} \in H, x^{*} \in F\left(u^{*}\right), y^{*} \in G\left(u^{*}\right), w^{*} \in S\left(u^{*}\right), g\left(u^{*}\right) \in C$ et $\rho>0$ tels que :

$$
\left\langle l, v-g\left(u^{*}\right)\right\rangle=\left(\frac{1}{2 r}\right)\|l\|\left\|v-g\left(u^{*}\right)\right\|^{2}, \quad \forall v \in C,
$$

où $l=h\left(y^{*}, g\left(w^{*}\right)\right)-\rho\left[T\left(x^{*}\right)-f\right]$. Si $l=0$, alors la preuve est terminée puisque on a toujours $0 \in N\left(C ; g\left(u^{*}\right)\right)$.

Supposons maintenant que $l \neq 0$. Alors (IVIN) upr $_{\text {implique }}$

$$
\left\langle\left(\frac{l}{\|l\|}\right), v-g\left(u^{*}\right)\right\rangle \leq\left\|v-g\left(u^{*}\right)\right\|^{2}, \quad \forall v \in C .
$$

Par conséquent, $\frac{l}{\|l\|} \in N\left(C ; g\left(u^{*}\right)\right)$, ce qui assure que

$$
0 \in l-N\left(C ; g\left(u^{*}\right)\right)=h\left(y^{*}, g\left(w^{*}\right)\right)-\rho\left[T\left(x^{*}\right)-f\right]-N\left(C ; g\left(u^{*}\right)\right) .
$$

Donc

$$
0 \in h\left(G\left(u^{*}\right), g\left(S\left(u^{*}\right)\right)\right)-\rho\left[T\left(x^{*}\right)-f\right]-N\left(C, g\left(u^{*}\right)\right) .
$$

Ceci termine la preuve de l'implication $(\Leftarrow)$.

$(\Rightarrow ?)$ Elle suit directement de la définition des ensembles uniformément prox-réguliers.

Remarque 2.5. Nous invitons le lecteur à lire les réfrerences $[5,10]$ pour un excellent exemple d'un problème d'élasto-plasticité dû à Panagiotopoulos et al. [10], que l'on peut reformuler sous la forme de notre problème (PVIN). 
Remarque 2.6. Le problème (PVIN) est considéré de manière qu'il soit le plus général possible et on peut avoir les exemples suivants comme cas particuliers :

(1) Soient $A, B: H \rightarrow H$ des applications telles que $: h(s, t)=B(s)-A(t)$, pour tout $s, t \in H$. Donc le problème (PVIN) s'écrit sous la forme suivante : trouver $u^{*} \in H$ et $\rho>0$, tels que :

$$
0 \in B\left(G\left(u^{*}\right)\right)-A\left(g\left(S\left(u^{*}\right)\right)\right)-\rho\left[T\left(F\left(u^{*}\right)\right)-f\right]-N\left(C, g\left(u^{*}\right)\right) .
$$

(2) Soient $F=G=S=i d$. Le problème (PVIN) s'écrit sous la forme suivante : trouver $u^{*} \in H$ et $\rho>0$, tels que :

$$
0 \in h\left(u^{*}, g\left(u^{*}\right)\right)-\rho\left[T\left(u^{*}\right)-f\right]-N\left(C, g\left(u^{*}\right)\right) .
$$

(3) Soient $F=G=S=i d$, et $h$ définie par $h(u, v)=A(u)-A(v)$, pour tout $u, v \in H$. Le problème (PVIN) s'écrit sous la forme suivante : trouver $u^{*} \in H$ et $\rho>0$, tels que :

$$
0 \in A\left(u^{*}\right)-A\left(g\left(u^{*}\right)\right)-\rho\left[T\left(u^{*}\right)-f\right]-N\left(C, g\left(u^{*}\right)\right) .
$$

(4) Soient $F=G=S=g=i d, h \equiv 0$ et $f=0$. Le problème (PVIN) s'écrit sous la forme suivante : trouver $u^{*} \in H$, tels que :

$$
T\left(u^{*}\right) \cap\left(-N\left(C, u^{*}\right)\right) \neq \emptyset .
$$

Ce problème (PVIN4) a été introduit et étudié dans [1]. Les problèmes (PVIN1)-(PVIN3) généralisent plusieurs types d'inégalités variationnelles introduites, étudiées et analysées par Noor [9], Zeng [14], Stampacchia [13] du cas convexe au cas non convexe et plus précisément aux cas uniformément prox-réguliers.

Dans toute la suite de cette section, nous supposons que $C$ est un sous-ensemble uniformément $r^{\prime}$-proxrégulier, avec $r^{\prime}>0$ et $\left.r \in\right] 0, r^{\prime}\left[\right.$. Nous notons $\zeta:=\frac{r^{\prime}}{r^{\prime}-r}$.

\section{1. $F$ est fortement monotone relativement à $T$}

Nous proposons l'algorithme suivant pour résoudre le problème (PVIN).

\section{Algorithme 2.7.}

Étape 1. Fixer $u_{0} \in H$ tel que $: g\left(u_{0}\right) \in C, x_{0} \in F\left(u_{0}\right), y_{0} \in G\left(u_{0}\right), w_{0} \in S\left(u_{0}\right)$ et $\rho>0$.

Étape 2: Pour $n \geq 0$. Calculer : $l_{n}=h\left(y_{n}, g\left(w_{n}\right)\right)-\rho\left[T\left(x_{n}\right)-f\right], z_{n+1}=g\left(u_{n}\right)+l_{n}$.

Choisir : $u_{n+1} \in H$ tel que $g\left(u_{n+1}\right) \in \operatorname{Proj}_{C}\left(z_{n+1}\right)$.

Choisir : $x_{n+1} \in F\left(u_{n+1}\right), y_{n+1} \in G\left(u_{n+1}\right)$ et $w_{n+1} \in S\left(u_{n+1}\right)$.

Pour analyser et étudier la convergence de cet algorithme, nous aurons besoin des hypothèses suivantes :

\section{Hypothèses (H1)}

(1) $F$ est $\alpha$-fortement monotone relativement $(\alpha>0)$ à $T$, c.-à-d., $\forall u_{1}, u_{2} \in H$ et $\forall x_{1} \in F\left(u_{1}\right), x_{2} \in F\left(u_{2}\right)$ on a

$$
\left\langle u_{1}-u_{2}, T\left(x_{1}\right)-T\left(x_{2}\right)\right\rangle \geq \alpha\left\|u_{1}-u_{2}\right\|^{2} .
$$

(2) $g$ est $\beta$-fortement monotone $(\beta>0)$, c.-à-d., $\forall u_{1}, u_{2} \in H$ on a

$$
\left\langle u_{1}-u_{2}, g\left(u_{1}\right)-g\left(u_{2}\right)\right\rangle \geq \beta\left\|u_{1}-u_{2}\right\|^{2} .
$$

(3) $h$ est $k_{1}$-lipschitzienne par rapport à la première variable et $k_{2}$-lipschitzienne par rapport à la deuxième variable, c.-à-d.,

$$
\begin{aligned}
\left\|h\left(y_{1}, z\right)-h\left(y_{2}, z\right)\right\| & \leq k_{1}\left\|y_{1}-y_{2}\right\|, \forall z, y_{1}, y_{2} \in H \\
\left\|h\left(y, z_{1}\right)-h\left(y, z_{2}\right)\right\| & \leq k_{2}\left\|z_{1}-z_{2}\right\|, \forall y, z_{1}, z_{2} \in H .
\end{aligned}
$$

(4) $G, S, F$ sont lipschitziennes au sens de Hausdorff avec des constantes $\eta, \lambda$ et $\gamma$ respectivement.

(5) $T$ et $g$ sont $\mu$-lipschitzienne et $\sigma$-lipschitzienne respectivement. 
(6) Le couple $(S, G)$ est $\theta$-fortement monotone relativement $(\theta>0)$ à $(h, g)$, c.-à-d., $\forall u_{1}, u_{2} \in H, y_{1} \in$ $G\left(u_{1}\right), y_{2} \in G\left(u_{2}\right), w_{1} \in S\left(u_{1}\right), w_{2} \in S\left(u_{2}\right)$ on a

$$
\left\langle h\left(y_{1}, g\left(w_{1}\right)\right)-h\left(y_{2}, g\left(w_{2}\right)\right), u_{1}-u_{2}\right\rangle \geq \theta\left\|u_{1}-u_{2}\right\|^{2} .
$$

(7) Les constantes $\alpha, \beta, \gamma, \mu, \eta, \sigma, k_{1}, k_{2}, \zeta$ et $\theta$ vérifient les inégalités suivantes :

- $\alpha^{2}>\mu^{2} \gamma^{2}-\mu^{2} \gamma^{2}\left[\frac{1-R}{\zeta}-R_{1}-\sigma\right]^{2}$.

- $R+\zeta\left(R_{1}+\sigma\right)<1$, avec $R:=\sqrt{1-2 \beta+\sigma^{2}}$ et $R_{1}:=\sqrt{1-2 \theta+\left(\eta k_{1}+\sigma \lambda k_{2}\right)^{2}}$.

Théorème 2.8. Supposons que toutes les hypothèses (H1) sont satisfaites et que le paramètre $\rho>0$ vérifie l'inégalité :

$$
\frac{\alpha}{\mu^{2} \gamma^{2}}-\epsilon<\rho<\min \left\{\frac{\alpha}{\mu^{2} \gamma^{2}}+\epsilon, \frac{r-\left\|h\left(y_{n}, g\left(u_{n}\right)\right)\right\|}{\left\|T\left(x_{n}\right)-f\right\|}\right\}
$$

où

$$
\epsilon=\frac{\sqrt{\alpha^{2}-\mu^{2} \gamma^{2}+\mu^{2} \gamma^{2}\left(\frac{1-R}{\zeta}-R_{1}-\sigma\right)^{2}}}{\mu^{2} \gamma^{2}} .
$$

Alors les suites $\left\{u_{n}\right\},\left\{x_{n}\right\},\left\{y_{n}\right\},\left\{w_{n}\right\}$ et $\left\{z_{n}\right\}$ engendrées par l'algorithme 2.7 sont fortement convergentes vers des éléments $u^{*}, x^{*}, y^{*}, w^{*}$ et $z^{*}$ dans $H$ respectivement, avec $u^{*}$ une solution de (PVIN).

Preuve. De l'algorithme 2.7 on a :

$$
\begin{gathered}
\left\|z_{n+1}-z_{n}\right\|=\left\|g\left(u_{n}\right)+h\left(y_{n}, g\left(w_{n}\right)\right)-\rho\left[T\left(x_{n}\right)-f\right]-g\left(u_{n-1}\right)-h\left(y_{n-1}, g\left(w_{n-1}\right)\right)+\rho\left[T\left(x_{n-1}\right)-f\right]\right\| \\
\leq\left\|g\left(u_{n}\right)-g\left(u_{n-1}\right)+u_{n}-u_{n-1}-\rho\left[T\left(x_{n}\right)-T\left(x_{n-1}\right)\right]\right\|+ \\
\left\|u_{n}-u_{n-1}-h\left(y_{n}, g\left(w_{n}\right)\right)-h\left(y_{n-1}, g\left(w_{n-1}\right)\right)\right\| .
\end{gathered}
$$

Comme $g$ est $\sigma$-lipschitzienne, on a :

$$
\left\|g\left(u_{n}\right)-g\left(u_{n-1}\right)\right\| \leq \sigma\left\|u_{n}-u_{n-1}\right\| .
$$

Il découle de la $\theta$-forte monotonie de $(S, G)$ relativement à $(h, g)$ :

$$
\left\langle h\left(y_{n}, g\left(w_{n}\right)\right)-h\left(y_{n-1}, g\left(w_{n-1}\right)\right), u_{n}-u_{n-1}\right\rangle \geq \theta\left\|u_{n}-u_{n-1}\right\|^{2} .
$$

La propriété de Lipschitz de $g, S$ et $G$, et la propriété de Lipschitz de $h$ par rapport aux deux variables donnent :

$$
\begin{aligned}
\left\|h\left(y_{n}, g\left(w_{n}\right)\right)+h\left(y_{n-1}, g\left(w_{n-1}\right)\right)\right\| & \leq k_{1}\left\|y_{n}-y_{n-1}\right\|+k_{2}\left\|g\left(w_{n}\right)-g\left(w_{n-1}\right)\right\| \\
& \leq k_{1} \eta\left\|u_{n}-u_{n-1}\right\|+k_{2} \sigma\left\|w_{n}-w_{n-1}\right\| \\
& \leq k_{1} \eta\left\|u_{n}-u_{n-1}\right\|+k_{2} \sigma \lambda\left\|u_{n}-u_{n-1}\right\| \\
& =\left(k_{1} \eta+k_{2} \sigma \lambda\right)\left\|u_{n}-u_{n-1}\right\| .
\end{aligned}
$$


D'où

$$
\begin{array}{r}
\left\|u_{n}-u_{n-1}-h\left(y_{n}, g\left(w_{n}\right)\right)+h\left(y_{n-1}, g\left(w_{n-1}\right)\right)\right\|^{2}=\left\|u_{n}-u_{n-1}\right\|^{2}-2\left\langle h\left(y_{n}, g\left(w_{n}\right)\right)-h\left(y_{n-1}, g\left(w_{n-1}\right)\right), u_{n}-u_{n-1}\right\rangle \\
+\left\|h\left(y_{n}, g\left(w_{n}\right)\right)-h\left(y_{n-1}, g\left(w_{n-1}\right)\right)\right\|^{2} \\
\leq\left\|u_{n}-u_{n-1}\right\|^{2}-2 \theta\left\|u_{n}-u_{n-1}\right\|^{2}+\left(k_{1} \eta+k_{2} \sigma \lambda\right)^{2}\left\|u_{n}-u_{n-1}\right\|^{2} \\
=\left[1-2 \theta+\left(k_{1} \eta+k_{2} \sigma \lambda\right)^{2}\right]\left\|u_{n}-u_{n-1}\right\|^{2}
\end{array}
$$

et donc

$$
\left\|u_{n}-u_{n-1}-h\left(y_{n}, g\left(w_{n}\right)\right)+h\left(y_{n-1}, g\left(w_{n-1}\right)\right)\right\| \leq R_{1}\left\|u_{n}-u_{n-1}\right\| .
$$

D'une part la $\alpha$-forte monotonie de $F$ relativement à $T$ donne :

$$
\left\langle u_{n}-u_{n-1}, T\left(x_{n}\right)-T\left(x_{n-1}\right)\right\rangle \geq \alpha\left\|u_{n}-u_{n-1}\right\|^{2},
$$

ce qui assure avec la propriété de Lipschitz de $T$ et la propriété de Lipschitz au sens de Hausdorffde de $F$ :

$$
\left\|u_{n}-u_{n-1}-\rho\left[T\left(u_{n}\right)-T\left(u_{n-1}\right)\right]\right\| \leq \sqrt{1-2 \rho \alpha+(\rho \mu \gamma)^{2}}\left\|u_{n}-u_{n-1}\right\|
$$

Ainsi on obtient

$$
\left\|z_{n+1}-z_{n}\right\| \leq m\left\|u_{n}-u_{n-1}\right\|
$$

avec

$$
m=\sigma+\sqrt{1-2 \theta+\left(\eta k_{1}+\sigma \lambda k_{2}\right)^{2}}+\sqrt{1-2 \rho \alpha+(\rho \mu \gamma)^{2}} .
$$

D'autre part et en utilisant l'hypothèse

$$
\rho<\frac{r-\left\|h\left(y_{n}, g\left(u_{n}\right)\right)\right\|}{\left\|T\left(x_{n}\right)-f\right\|}
$$

on obtient pour tout $n$

$$
\begin{aligned}
d\left(C, z_{n+1}\right) & =d\left(C, g\left(u_{n}\right)+h\left(y_{n}, g\left(w_{n}\right)\right)-\rho\left[T\left(x_{n}\right)-f\right]\right)-d\left(C, g\left(u_{n}\right)\right) \\
& \leq\left\|h\left(y_{n}, g\left(w_{n}\right)\right)-\rho\left[T\left(x_{n}\right)-f\right]\right\| \\
& \leq\left\|h\left(y_{n}, g\left(w_{n}\right)\right)\right\|+\rho\left\|T\left(x_{n}\right)-f\right\| \\
& <\left\|h\left(y_{n}, g\left(w_{n}\right)\right)\right\|+\frac{r-\left\|h\left(y_{n}, g\left(u_{n}\right)\right)\right\|}{\left\|T\left(x_{n}\right)-f\right\|}\left\|T\left(x_{n}\right)-f\right\|=r
\end{aligned}
$$

ce qui assure que la suite $\left\{z_{n}\right\}_{n}$ reste dans l'élargissement $C(r)$ de l'ensemble $C$. Par conséquent la proposition 1.8-(3), l'uniforme prox-régularité de l'ensemble $C$, et la forte monotonie de $g$ assurent :

$$
\begin{aligned}
\left\|u_{n+1}-u_{n}\right\| & \leq\left\|u_{n+1}-u_{n}-\left[g\left(u_{n+1}\right)-g\left(u_{n}\right)\right]\right\|+\left\|g\left(u_{n+1}\right)-g\left(u_{n}\right)\right\| \\
& \leq R\left\|u_{n+1}-u_{n}\right\|+\left\|\operatorname{Proj}_{C}\left(z_{n+1}\right)-\operatorname{Proj}_{C}\left(z_{n}\right)\right\| \\
& \leq R\left\|u_{n+1}-u_{n}\right\|+\zeta\left\|z_{n+1}-z_{n}\right\| .
\end{aligned}
$$

D'où

$$
\left\|u_{n+1}-u_{n}\right\| \leq \frac{\zeta}{1-R}\left\|z_{n+1}-z_{n}\right\| \leq \frac{m \zeta}{1-R}\left\|u_{n+1}-u_{n}\right\| .
$$

D'après l'hypothèse $(7)$ dans $(\mathrm{H} 1)$ la constante $\frac{m \zeta}{1-R}$ est toujours strictement inférieure à 1 . La suite $\left\{u_{n}\right\}_{n}$ est alors de Cauchy, donc convergente vers un élément $u^{*} \in H$. Par construction des suites $\left\{x_{n}\right\}_{n},\left\{y_{n}\right\}_{n}$ et $\left\{w_{n}\right\}_{n}$ 
et en utilisant la propriéte de Lipschitz au sens de Hausdorff de $F, G$ et de $S$ on obtient la convergence de ces suites vers des éléments $x^{*}, y^{*}$ et $w^{*}$ respectivement, avec

$$
x^{*} \in F\left(u^{*}\right), y^{*} \in G\left(u^{*}\right) \text { et } w^{*} \in S\left(u^{*}\right) .
$$

Il suit de l'algorithme 2.7, de la convergence des suites $\left\{x_{n}\right\}_{n},\left\{y_{n}\right\}_{n}$ et $\left\{w_{n}\right\}_{n}$, et de la continuité de $h, g, T, S, F$ et $G$, que la suite $\left\{z_{n}\right\}_{n}$ est convergente vers un élément $z^{*} \in H$ vérifiant

$$
z^{*}=g\left(u^{*}\right)+h\left(y^{*}, g\left(w^{*}\right)\right)-\rho\left[T\left(x^{*}\right)-f\right] .
$$

Maintenant, on doit montrer que $u^{*}$ est une solution de (PVIN). Par construction on a :

$$
g\left(u_{n+1}\right) \in \operatorname{Proj}_{C}\left(z_{n+1}\right) .
$$

Donc, la définition du cône normal proximal donne

$$
z_{n+1}-g\left(u_{n+1}\right) \in N^{P}\left(C, g\left(u_{n+1}\right)\right), \forall n \geq 0
$$

et comme $\left\{z_{n}\right\}_{n}$ et $\left\{g\left(u_{n}\right)\right\}_{n}$ convergent vers $z^{*}, g\left(u^{*}\right)$ respectivement et d'après la proposition 1.11, on peut passer à la limite dans la dernière relation et on obtient

$$
z^{*}-g\left(u^{*}\right) \in N\left(C, g\left(u^{*}\right)\right), \text { i.e., } h\left(y^{*}, g\left(w^{*}\right)\right)-\rho\left[T\left(x^{*}\right)-f\right] \in N\left(C, g\left(u^{*}\right)\right),
$$

ce qui assure que $u^{*}$ est une solution de (PVIN).

\subsection{F n'est pas nécessairement fortement monotone relativement à $T$}

Dans cette section, nous allons montrer que les résultats obtenus dans le théorème 2.8 peuvent être étendus au cas où $F=F_{1}+F_{2}$, avec $F_{1}$ est lipschitzienne au sens de Hausdorff, fortement monotone relativement à $T$ dans $H$, et $F_{2}$ est seulement lipschitzienne au sens de Hausdorff dans $H$. Notons que dans ce cas $F$ n'est pas nécessairement fortement monotone relativement à $T$.

Nous proposons l'algorithme suivant pour résoudre le problème (PVIN) associé à la multifonction $F=$ $F_{1}+F_{2}:$

\section{Algorithme 2.9.}

Étape 1. Fixer $\rho>0$ et $u_{0} \in H$ tels que

$$
g\left(u_{0}\right) \in C, x_{0}^{1} \in F_{1}\left(u_{0}\right), x_{0}^{2} \in F_{2}\left(u_{0}\right), y_{0} \in G\left(u_{0}\right), w_{0} \in S\left(u_{0}\right) .
$$

Étape 2. Pour tout $n \geq 0$ :

Calculer $l_{n}=h\left(y_{n}, g\left(w_{n}\right)\right)-\rho\left[T\left(x_{n}^{1}\right)+T\left(x_{n}^{2}\right)-f\right]$ et $z_{n+1}=g\left(u_{n}\right)+l_{n}$.

Choisir : $u_{n+1} \in H$ tel que $g\left(u_{n+1}\right) \in \operatorname{Proj}_{C}\left(z_{n+1}\right)$.

Choisir : $x_{n+1}^{k} \in F_{k}\left(u_{n+1}\right),(k=1,2) y_{n+1} \in G\left(u_{n+1}\right)$, et $w_{n+1} \in S\left(u_{n+1}\right)$.

\section{Hypothèses (H2) :}

(1) $F_{1}$ est $\alpha_{1}$-fortement monotone relativement à $T$ et $\gamma_{1}$-lipschitzienne au sens de Hausdorff.

(2) $F_{1}$ et $F_{2}$ sont lipschitziennes au sens de Hausdorff avec des constantes $\gamma_{1}, \gamma_{2}$ respectivement.

(3) Les constantes $\alpha_{1}, \beta, \eta, \lambda, \mu, \sigma, \gamma_{1}, \gamma_{2}, k_{1}, k_{2}$, et $\theta$ vérifient les inégalités suivantes :

- $\gamma_{1}>\gamma_{2}$;

- $\alpha_{1}^{2}>\mu^{2}\left(\gamma_{1}^{2}-\gamma_{2}^{2}\right)+2 \mu \alpha_{1} \gamma_{2}\left(\frac{1-R}{\zeta}-\sigma-R_{1}\right)$. 
Théorème 2.10. Supposons que les hypothèses (2)-(6) dans (H1) et toutes les hypothèses (H2) sont satisfaites et que le paramètre $\rho>0$ vérifie les inégalités :

$$
\begin{gathered}
\rho>\frac{\alpha_{1} \zeta-\mu \gamma_{2}\left(1-R-\sigma \zeta-R_{1} \zeta\right)}{\mu^{2} \zeta\left(\gamma_{1}^{2}-\gamma_{2}^{2}\right)}-\epsilon, \\
\rho<\min \left\{\frac{\alpha_{1} \zeta-\mu \gamma_{2}\left(1-R-\sigma \zeta-R_{1} \zeta\right)}{\mu^{2} \zeta\left(\gamma_{1}^{2}-\gamma_{2}^{2}\right)}+\epsilon, \frac{\left(1-R-\sigma \zeta-R_{1} \zeta\right)}{\mu \gamma_{2} \zeta}, \frac{r-\left\|h\left(y_{n}, g\left(u_{n}\right)\right)\right\|}{\left\|T\left(x_{n}^{1}\right)+T\left(x_{n}^{2}\right)-f\right\|}\right\}
\end{gathered}
$$

où

$$
\epsilon=\frac{\sqrt{\alpha_{1}^{2}-\mu^{2}\left(\gamma_{1}^{2}-\gamma_{2}^{2}\right)+\mu^{2} \gamma_{1}^{2}\left(\frac{1-R}{\zeta}-R_{1}-\sigma\right)^{2}-2 \alpha_{1} \gamma_{2}\left(\frac{1-R}{\zeta}-R_{1}-\sigma\right)}}{\zeta \mu^{2}\left(\gamma_{1}^{2}-\gamma_{2}^{2}\right)} .
$$

Alors les suites $\left\{u_{n}\right\},\left\{x_{n}^{1}\right\},\left\{x_{n}^{2}\right\},\left\{y_{n}\right\},\left\{w_{n}\right\}$, et $\left\{z_{n}\right\}$ engendrées par l'algorithme 2.9 sont fortement convergentes vers des éléments $u^{*}, x^{1}, x^{2}, y^{*}, w^{*}$ et $z^{*}$ dans $H$ respectivement, avec $u^{*}$ une solution de (PVIN) associée $\grave{a} F=F_{1}+F_{2}$.

Preuve. On suit la démonstration du théorème 2.8 avec des petites modifications. De l'algorithme 2.9 on a :

$$
\begin{aligned}
\left\|z_{n+1}-z_{n}\right\| \leq & \| g\left(u_{n}\right)+h\left(y_{n}, g\left(w_{n}\right)\right)-\rho\left[T\left(x_{n}^{1}\right)+T\left(x_{n}^{2}\right)-f\right] \\
& -g\left(u_{n-1}\right)-h\left(y_{n-1}, g\left(w_{n-1}\right)\right)+\rho\left[T\left(x_{n-1}^{1}\right)+T\left(x_{n-1}^{2}\right)-f\right] \| \\
\leq & \left\|g\left(u_{n}\right)-g\left(u_{n-1}\right)\right\|+\left\|u_{n}-u_{n-1}-h\left(y_{n}, g\left(w_{n}\right)\right)-h\left(y_{n-1}, g\left(w_{n-1}\right)\right)\right\| \\
& +\rho\left\|T\left(x_{n-1}^{2}\right)-T\left(x_{n}^{2}\right)\right\|+\left\|u_{n}-u_{n-1}-\rho\left[T\left(x_{n}^{1}\right)-T\left(x_{n-1}^{1}\right)\right]\right\| .
\end{aligned}
$$

De la même manière que dans la preuve du théorème 2.8 on obtient

$$
\left\|u_{n}-u_{n-1}-h\left(y_{n}, g\left(w_{n}\right)\right)-h\left(y_{n-1}, g\left(w_{n-1}\right)\right)\right\| \leq R_{1}\left\|u_{n}-u_{n-1}\right\| .
$$

D'autre part la $\alpha_{1}$-forte monotonie de $F_{1}$ relativement à $T$, et la propriété de Lipschitz au sens de Hausdorff de $F_{1}, F_{2}$ et de $T$, donnent :

- $\rho\left\|T\left(x_{n}^{2}\right)-T\left(x_{n-1}^{2}\right)\right\| \leq \rho \mu\left\|u_{n}-u_{n-1}\right\| ;$

- $\left\|\left(u_{n}-u_{n-1}\right)-\rho\left[T\left(x_{n}^{1}\right)-T\left(x_{n-1}^{1}\right)\right]\right\| \leq \sqrt{1-2 \rho \alpha_{1}+\left(\rho \mu \gamma_{1}\right)^{2}}\left\|u_{n}-u_{n-1}\right\|$.

Finalement on obtient :

$$
\left\|z_{n+1}-z_{n}\right\| \leq m\left\|u_{n}-u_{n-1}\right\|
$$

avec

Par hypothèse on a :

$$
m=\left[\sigma+R_{1}+\rho \mu \gamma_{2}+\sqrt{1-2 \rho \alpha_{1}+\left(\rho \mu \gamma_{1}\right)^{2}}\right] .
$$

$$
\rho<\frac{r-\left\|h\left(y_{n}, g\left(u_{n}\right)\right)\right\|}{\left\|T\left(x_{n}^{1}\right)+T\left(x_{n}^{2}\right)-f\right\|},
$$

d'où $d\left(C, z_{n+1}\right)<r$, ce qui assure, que la suite $\left\{z_{n}\right\}$ reste dans l'ensemble $C(r)$. Par conséquent la proposition 1.8-(3), l'uniforme prox-régularité de l'ensemble $C$ et la forte monotonie de $g$ assurent :

$$
\begin{aligned}
\left\|u_{n+1}-u_{n}\right\| & \leq\left\|u_{n+1}-u_{n}-g\left(u_{n+1}\right)+g\left(u_{n}\right)\right\|+\left\|g\left(u_{n+1}\right)-g\left(u_{n}\right)\right\| \\
& \leq R\left\|u_{n+1}-u_{n}\right\|+\left\|\operatorname{Proj}_{C}\left(z_{n+1}\right)-\operatorname{Proj}_{C}\left(z_{n}\right)\right\| \\
& \leq R\left\|u_{n+1}-u_{n}\right\|+\zeta\left\|z_{n+1}-z_{n}\right\| \\
& \leq R\left\|u_{n+1}-u_{n}\right\|+\zeta m\left\|u_{n}-u_{n-1}\right\|
\end{aligned}
$$


d'où

$$
\left\|u_{n+1}-u_{n}\right\| \leq \frac{\zeta m}{1-R}\left\|u_{n}-u_{n-1}\right\|
$$

D'après l'hypothèse $(3)$ dans (H2), la constante $\frac{\zeta m}{1-R}$ est toujours strictement inférieure à 1 . La suite $\left\{u_{n}\right\}$ est alors de Cauchy, donc elle converge vers un élément $u^{*} \in H$.

La construction des suites $\left\{x_{n}^{1}\right\},\left\{x_{n}^{2}\right\},\left\{y_{n}\right\}$ et $\left\{w_{n}\right\}$, avec la propriété de Lipschitz au sens de Hausdorff de $F_{1}, F_{2}, G$ et de $S$ assurent la convergence de ces suites vers des éléments $x_{1}, x_{2}, y^{*}$ et $w^{*}$ avec $x^{1} \in F_{1}\left(u^{*}\right)$, $x^{2} \in F_{2}\left(u^{*}\right), y^{*} \in G\left(u^{*}\right)$ et $w^{*} \in S\left(u^{*}\right)$. Il suit donc de l'algorithme 2.9, de la convergence des suites $\left\{x_{n}^{1}\right\},\left\{x_{n}^{2}\right\}$, $\left\{y_{n}\right\}$ et $\left\{w_{n}\right\}$ et de la continuité de $h, g, T, G, S, F_{1}$ et $F_{2}$, que la suite $z_{n}$ est aussi convergente vers l'élément

$$
z^{*}=g\left(u^{*}\right)+h\left(y^{*}, g\left(w^{*}\right)\right)-\rho\left[T\left(x_{1}\right)+T\left(x_{2}\right)-f\right]
$$

Maintenant, on doit montrer que $u^{*}$ est une solution de (PVIN) associée à la multifonction $F=F_{1}+F_{2}$. Par construction, on a $g\left(u_{n+1}\right) \in \operatorname{Proj}_{C}\left(z_{n+1}\right), \forall n \geq 0$. Donc

$$
z_{n+1}-g\left(u_{n+1}\right) \in N\left(C, g\left(u_{n+1}\right)\right), \quad \forall n \geq 0 .
$$

Comme $\left\{z_{n}\right\}$ et $\left\{g\left(u_{n}\right)\right\}$ convergent vers $z^{*}$ et $g\left(u^{*}\right)$ respectivement, et d'après la proposition 1.11 et la remarque 1.12, on peut passer à la limite dans la dernière relation, et on obtient :

$$
z^{*}-g\left(u^{*}\right) \in N\left(C, g\left(u^{*}\right)\right) \text {, c.-à-d. } h\left(y^{*}, g\left(w^{*}\right)\right)-\rho\left[T\left(x^{1}\right)+T\left(x^{2}\right)-f\right] \in N\left(C, g\left(u^{*}\right)\right) \text {, }
$$

et puisque $x^{1} \in F_{1}\left(u^{*}\right), x^{2} \in F_{2}\left(u^{*}\right), y^{*} \in G\left(u^{*}\right)$ et $w^{*} \in S\left(u^{*}\right)$ on obtient

$$
0 \in\left\{h\left(G\left(u^{*}\right), g\left(S\left(u^{*}\right)\right)\right)-\rho\left[T\left(F_{1}\left(u^{*}\right)+F_{2}\left(u^{*}\right)\right)-f\right]-N\left(C, g\left(u^{*}\right)\right)\right\} .
$$

D'où, $u^{*}$ est une solution de (PVIN) associée à la multifonction $F=F_{1}+F_{2}$.

\section{EXTENSION}

Dans cette section nous allons modifier le problème (PVIN) pour englober plus de cas particuliers. Cette fois-ci nous allons supposer que l'ensemble $C$ est une multifonction définie de $H$ dans $H$. Le problème (PVIN) devient :

$$
\begin{aligned}
& \text { trouver } u^{*} \in H \text { et } \rho>0 \text { tels que } g\left(u^{*}\right) \in C\left(u^{*}\right) \text { et } \\
& \qquad 0 \in\left\{h\left(G\left(u^{*}\right), g\left(S\left(u^{*}\right)\right)\right)-\rho\left[T\left(F\left(u^{*}\right)\right)-f\right]-N\left(C\left(u^{*}\right), g\left(u^{*}\right)\right)\right\} .
\end{aligned}
$$

Ce problème est appelé Problème Quasi-Variationnel Implicite et Nonlinéaire (PQVIN).

Dans toute la suite de cette section, nous allons supposer que $C$ est une multifonction à valeurs fermées non vides et $r^{\prime}$-prox-régulière avec $r^{\prime}>0$. Nous allons aussi fixer $\left.r \in\right] 0, r^{\prime}\left[\right.$ et $\zeta=\frac{r^{\prime}}{r^{\prime}-r}$.

\subsection{F est fortement monotone relativement à $T$}

Nous proposons l'algorithme suivant pour résoudre le problème (PQVIN).

\section{Algorithme 3.1.}

Étape 1. Fixer $u_{0} \in H$ et $\rho>0$, tel que :

$$
g\left(u_{0}\right) \in C\left(u_{0}\right), x_{0} \in F\left(u_{0}\right), y_{0} \in G\left(u_{0}\right), w_{0} \in S\left(u_{0}\right) .
$$


Étape 2. Pour $n \geq 0$ : Calculer : $l_{n}=h\left(y_{n}, g\left(w_{n}\right)\right)-\rho\left[T\left(x_{n}\right)-f\right], z_{n+1}=g\left(u_{n}\right)+l_{n}$.

Choisir : $u_{n+1} \in H$ tel que $: g\left(u_{n+1}\right) \in \operatorname{Proj}_{C\left(u_{n}\right)}\left(z_{n+1}\right)$.

Choisir : $x_{n+1} \in F\left(u_{n+1}\right), y_{n+1} \in G\left(u_{n+1}\right), w_{n+1} \in S\left(u_{n+1}\right)$.

\section{Hypothèses (H3)}

(1) $C$ est $\kappa$-lipschitzienne avec $0<\kappa<1$.

(2) La projection vérifie l'inégalité suivante :

$$
\left\|\operatorname{Proj}_{C(x)}(z)-\operatorname{Proj}_{C(y)}(z)\right\| \leq k\|x-y\| \quad \forall x, y, z \in H, \text { avec } 0<k<1 .
$$

(3) Soit $a$ vérifiant :

$$
0<a<\left[\frac{r(1-\kappa-R)}{1+3 \kappa-R}\right] .
$$

(4) Les constantes $\alpha, \beta, \gamma, \lambda, \mu, \eta, \sigma, k, k_{1}, k_{2}, \zeta$ et $\theta$ vérifient les inégalités suivantes :

$$
\begin{aligned}
& \text { - } \alpha^{2}>\mu^{2} \gamma^{2}-\mu^{2} \gamma^{2}\left[\frac{1-R-k}{\zeta}-\sigma-R_{1}\right]^{2} ; \\
& \text { - } k+R+\zeta\left(R_{1}+\sigma\right)<1 .
\end{aligned}
$$

Théorème 3.2. Supposons que les hypothèses (1)-(6) dans (H1) et toutes les hypothèses (H3) sont satisfaites et que le paramètre $\rho>0$ vérifie l'inégalité :

$$
\frac{\alpha}{\mu^{2} \gamma^{2}}-\epsilon<\rho<\min \left\{\frac{\alpha}{\mu^{2} \gamma^{2}}+\epsilon, \frac{a-\left\|h\left(y_{n}, g\left(u_{n}\right)\right)\right\|}{\left\|T\left(x_{n}\right)-f\right\|}\right\},
$$

où

$$
\epsilon=\frac{\sqrt{\alpha^{2}-\mu^{2} \gamma^{2}+\frac{\mu^{2} \gamma^{2}}{\zeta^{2}}\left[1-k-R-\zeta\left(\sigma+R_{1}\right)\right]^{2}}}{\mu^{2} \gamma^{2}} .
$$

Alors les suites $\left\{u_{n}\right\},\left\{x_{n}\right\},\left\{y_{n}\right\},\left\{w_{n}\right\}$, et $\left\{z_{n}\right\}$ engendrées par l'algorithme 3.4 sont fortement convergentes vers des éléments $u^{*}, x^{*}, y^{*}, w^{*}$ et $z^{*}$ dans $H$ respectivement, avec $u^{*}$ une solution de (PQVIN).

Nous commençons tout d'abord par prouver le lemme suivant qui est un point clé de la preuve du théorème 3.2.

Lemme 3.3. Sous les mêmes hypothèses du théorème 3.2, les suites $\left\{u_{n}\right\},\left\{z_{n}\right\}$, engendrées par l'algorithme 3.4 vérifient pour tout $n \geq 0$

$$
z_{n+1} \text { et } z_{n} \in\left[C\left(u_{n}\right)\right]_{r}:=\left\{y \in H ; d\left(C\left(u_{n}\right), y\right)<r\right\} .
$$

Preuve. Observons tout d'abord que les hypothèses faites sur le paramètre $\rho$ dans le théorème 3.2 assurent que pour tout $n \geq 0$

$$
\left\|l_{n}\right\|=\| h\left(y_{n}, g\left(w_{n}\right)\right)-\rho\left[T\left(x_{n}\right)-f\|\leq\| h\left(y_{n}, g\left(w_{n}\right)\right)\|-\rho\| T\left(x_{n}\right)-f \|<a .\right.
$$

Maintenant, de l'algorithme 3.4 on a

- Pour $n=0$,

$$
d\left(C\left(u_{0}\right), z_{1}\right)=d\left(C\left(u_{0}\right), g\left(u_{0}\right)+l_{0}\right) \leq d\left(C\left(u_{0}\right), g\left(u_{0}\right)\right)+\left\|l_{0}\right\|=\left\|l_{0}\right\|<a .
$$

- Pour $n=1$,

$$
\begin{aligned}
d\left(C\left(u_{1}\right), z_{2}\right) & =d\left(C\left(u_{1}\right), g\left(u_{1}\right)+l_{1}\right)-d\left(C\left(u_{0}\right), g\left(u_{1}\right)\right) \\
& \leq\left\|l_{1}\right\|+\kappa\left\|u_{1}-u_{0}\right\| \\
& \leq a+\kappa\left\|u_{1}-u_{0}\right\|
\end{aligned}
$$


et

$$
d\left(C\left(u_{1}\right), z_{1}\right) \leq d\left(C\left(u_{0}\right), z_{1}\right)+\kappa\left\|u_{1}-u_{0}\right\|<a+\kappa\left\|u_{1}-u_{0}\right\|
$$

D'un autre côté on a

$$
\left\|u_{1}-u_{0}\right\| \leq\left\|u_{1}-u_{0}+g\left(u_{1}\right)-g\left(u_{0}\right)\right\|+\left\|g\left(u_{1}\right)-z_{1}\right\|+\left\|g\left(u_{0}\right)-z_{1}\right\| .
$$

La forte monotonie de $g$ et sa proprieté de Lipschitz donnent

$$
\left\|u_{1}-u_{0}+g\left(u_{1}\right)-g\left(u_{0}\right)\right\|^{2} \leq\left\|u_{1}-u_{0}\right\|^{2}+\sigma^{2}\left\|u_{1}-u_{0}\right\|^{2}-2 \beta\left\|u_{1}-u_{0}\right\|^{2} \leq\left(1-2 \beta+\sigma^{2}\right)\left\|u_{1}-u_{0}\right\|^{2}
$$

D'où

$$
\left\|u_{1}-u_{0}\right\| \leq R\left\|u_{1}-u_{0}\right\|+d\left(C\left(u_{0}\right), z_{1}\right)+\left\|l_{0}\right\| \leq R\left\|u_{1}-u_{0}\right\|+2 a,
$$

et puisque $0<R<1$ on obtient $\left\|u_{1}-u_{0}\right\|<\frac{2 a}{1-R}$.

Par conséquent

$$
a+\kappa\left\|u_{1}-u_{0}\right\|<a+\kappa \frac{2 a}{1-R}=a\left(1+\frac{2 \kappa}{1-R}\right)<r\left(\frac{1-\kappa-R}{1+3 \kappa-R}\right)\left(\frac{1-R+2 \kappa}{1-R}\right)<r,
$$

ce qui assure que $d\left(C\left(u_{1}\right), z_{2}\right)$ et $d\left(C\left(u_{1}\right), z_{1}\right)$ sont inférieurs à $r$. Donc la propriété est vérifiée pour $n=1$.

- Pour $n \geq 2$, on a

$$
\begin{aligned}
d\left(C\left(u_{n}\right), z_{n+1}\right) & =d\left(C\left(u_{n}\right), g\left(u_{n}\right)+l_{n}\right) \\
& \leq d\left(C\left(u_{n}\right), g\left(u_{n}\right)\right)+\left\|l_{n}\right\|-d\left(C\left(u_{n-1}\right), g\left(u_{n}\right)\right) \\
& \leq a+\kappa\left\|u_{n}-u_{n-1}\right\|
\end{aligned}
$$

ce qui donne

$$
\begin{aligned}
d\left(C\left(u_{n}\right), z_{n}\right) & \leq d\left(C\left(u_{n-1}\right), z_{n}\right)+\kappa\left\|u_{n}-u_{n-1}\right\| \\
& \leq a+\kappa\left\|u_{n-1}-u_{n-2}\right\|+\kappa\left\|u_{n}-u_{n-1}\right\|
\end{aligned}
$$

D'une autre part on a

$$
\left\|u_{n}-u_{n-1}\right\| \leq\left\|u_{n}-u_{n-1}+g\left(u_{n}\right)-g\left(u_{n-1}\right)\right\|+\left\|g\left(u_{n}\right)-z_{n}\right\|+\left\|g\left(u_{n-1}\right)-z_{n}\right\| .
$$

La forte monotonie de $g$ et sa proprieté de Lipschitz donnent

$$
\begin{aligned}
\left\|u_{n}-u_{n-1}+g\left(u_{n}\right)-g\left(u_{n-1}\right)\right\|^{2} & \leq\left\|u_{n}-u_{n-1}\right\|^{2}+\sigma^{2}\left\|u_{n}-u_{n-1}\right\|^{2}-2 \beta\left\|u_{n}-u_{n-1}\right\|^{2} \\
& =\left(1-2 \beta+\sigma^{2}\right)\left\|u_{n}-u_{n-1}\right\|^{2} .
\end{aligned}
$$

D'où

$$
\left\|u_{n}-u_{n-1}\right\| \leq R\left\|u_{n}-u_{n-1}\right\|+d\left(C\left(u_{n-1}\right), z_{n}\right)+\left\|l_{n-1}\right\| \leq R\left\|u_{n}-u_{n-1}\right\|+2 a+\kappa\left\|u_{n-1}-u_{n-2}\right\|,
$$

et puisque $0<R<1$ on obtient

$$
\left\|u_{n}-u_{n-1}\right\| \leq \frac{2 a+\kappa\left\|u_{n-1}-u_{n-2}\right\|}{1-R}
$$


d'où

Et on obtient finalement

$$
\begin{aligned}
(1-R)\left\|u_{n}-u_{n-1}\right\| & \leq 2 a+\left(\frac{\kappa}{1-R}\right)\left(2 a+\kappa\left\|u_{n-2}-u_{n-3}\right\|\right) \\
& \leq 2 a+2 a\left(\frac{\kappa}{1-R}\right)+\left(\frac{\kappa}{1-R}\right)^{2}\left[2 a+\kappa\left\|u_{n-3}-u_{n-4}\right\|\right] \\
& \leq 2 a+2 a\left(\frac{\kappa}{1-R}\right)+2 a\left(\frac{\kappa}{1-R}\right)^{2}+\left(\frac{\kappa}{1-R}\right)^{3}\left[2 a+\kappa\left\|u_{n-4}-u_{n-5}\right\|\right] .
\end{aligned}
$$

$$
\left\|u_{n}-u_{n-1}\right\| \leq\left(\frac{2 a}{1-R}\right)\left[1+\frac{\kappa}{1-R}+\left(\frac{\kappa}{1-R}\right)^{2}+\cdots+\left(\frac{\kappa}{1-R}\right)^{n-2}\left(1+\frac{\kappa\left\|u_{1}-u_{0}\right\|}{2 a}\right)\right] .
$$

Puisque $\left\|u_{1}-u_{0}\right\|<\frac{2 a}{1-R}$ on obtient $\frac{\kappa\left\|u_{1}-u_{0}\right\|}{2 a}<\frac{\kappa}{1-R}$, d'où

$$
\begin{aligned}
\left\|u_{n}-u_{n-1}\right\| & \leq\left(\frac{2 a}{1-R}\right)\left[1+\frac{\kappa}{1-R}+\left(\frac{\kappa}{1-R}\right)^{2}+\cdots+\left(\frac{\kappa}{1-R}\right)^{n-2}+\left(\frac{\kappa}{1-R}\right)^{n-1}\right] \\
& \leq\left(\frac{2 a}{1-R}\right) \sum_{p=0}^{n-1}\left(\frac{\kappa}{1-R}\right)^{p} \\
& \leq\left(\frac{2 a}{1-R}\right) \frac{1-\left(\frac{\kappa}{1-R}\right)^{n}}{1-\left(\frac{\kappa}{1-R}\right)} \\
& \leq 2 a \frac{\left[1-\left(\frac{\kappa}{1-R}\right)^{n}\right]}{1-R-\kappa}<\frac{2 a}{1-R-\kappa} .
\end{aligned}
$$

Par conséquent des simples calculs donnent

$$
d\left(C\left(u_{n}\right), z_{n+1}\right) \leq \frac{a}{1-R-\kappa}[1-R+\kappa]<r
$$

et

$$
d\left(C\left(u_{n}\right), z_{n}\right) \leq \frac{a}{1-R-\kappa}[1-R+3 \kappa]<r,
$$

ce qui assure que $z_{n+1}$ et $z_{n} \in\left[C\left(u_{n}\right)\right]_{r}, \forall n \geq 0$.

Revenons maintenant à la preuve du théorème 3.2.

Preuve. En suivant la même démarche dans la preuve du théorème 2.8 on obtient

$$
\left\|z_{n+1}-z_{n}\right\| \leq m\left\|u_{n}-u_{n-1}\right\|,
$$

avec

$$
m=\left[\sigma+R_{1}+\sqrt{1-2 \rho \alpha+(\rho \mu \gamma)^{2}}\right] .
$$

D'après le lemme 3.3 on a $z_{n+1}$ et $z_{n} \in\left[C\left(u_{n}\right)\right]_{r}, \forall n \geq 0$. Par conséquent la proposition 1.10 et nos hypothèses du théorème assurent

$$
\begin{aligned}
\left\|u_{n+1}-u_{n}\right\|= & \left\|u_{n+1}-u_{n}-\left[g\left(u_{n+1}\right)-g\left(u_{n}\right)\right]\right\|+\left\|g\left(u_{n+1}\right)-g\left(u_{n}\right)\right\| \\
\leq & \sqrt{1-2 \beta+\sigma^{2}}\left\|u_{n+1}-u_{n}\right\|+\left\|\operatorname{Proj}_{C\left(u_{n}\right)}\left(z_{n+1}\right)-\operatorname{Proj}_{C\left(u_{n-1}\right)}\left(z_{n}\right)\right\| \\
\leq & R\left\|u_{n+1}-u_{n}\right\|+\left\|\operatorname{Proj}_{C\left(u_{n}\right)}\left(z_{n+1}\right)-\operatorname{Proj}_{C\left(u_{n}\right)}\left(z_{n}\right)\right\| \\
& +\left\|\operatorname{Proj}_{C\left(u_{n}\right)}\left(z_{n}\right)-\operatorname{Proj}_{C\left(u_{n-1}\right)}\left(z_{n}\right)\right\| \\
\leq & R\left\|u_{n+1}-u_{n}\right\|+\zeta\left\|z_{n+1}-z_{n}\right\|+k\left\|u_{n}-u_{n-1}\right\| .
\end{aligned}
$$


D'où

et puisque $0<R<1$ on obtient

$$
(1-R)\left\|u_{n+1}-u_{n}\right\| \leq(k+m \zeta)\left\|u_{n}-u_{n-1}\right\|,
$$

$$
\left\|u_{n+1}-u_{n}\right\| \leq \frac{k+m \zeta}{1-R}\left\|u_{n}-u_{n-1}\right\|
$$

où $m=\sigma+R_{1}+\sqrt{1-2 \rho \alpha+(\rho \mu \gamma)^{2}}$.

D'après (4) dans (H3) la constante $\frac{k+m \zeta}{1-R}$ est toujours strictement inférieure à 1. D'où la convergence forte de la suite $\left\{u_{n}\right\}$ vers un élément $u^{*}$, ainsi que $\left\{x_{n}\right\},\left\{y_{n}\right\}$ et $\left\{w_{n}\right\}$ vers des éléments $x^{*}, y^{*}$ et $w^{*}$ dans $H$ respectivement. La continuité des applications $h, T$ et des multifonctions $F, G, S$ donne :

$$
x^{*} \in F\left(u^{*}\right), y^{*} \in G\left(u^{*}\right) \text { et } w^{*} \in S\left(u^{*}\right),
$$

et donne aussi la convergence forte de la suite $\left\{z_{n}\right\}$ vers l'élément $z^{*}$ donné par

$$
z^{*}=g\left(u^{*}\right)+h\left(y^{*}, g\left(w^{*}\right)\right)-\rho\left[T\left(x^{*}\right)-f\right] .
$$

Maintenant, on doit montrer que $u^{*}$ est une solution du (PQVIN). Premièrement, d'après la propriété de Lipschitz de $g$ et de $C$ on a $: g\left(u^{*}\right) \in C\left(u^{*}\right)$. D'autre part on a par construction $g\left(u_{n+1}\right)=\operatorname{Proj}_{C\left(u_{n}\right)}\left(z_{n+1}\right), \forall n \geq$ 0 . Donc, d'après la définition du cône normal proximal on obtient

$$
z_{n+1}-g\left(u_{n+1}\right) \in N^{P}\left(C\left(u_{n}\right), g\left(u_{n+1}\right)\right), \quad \forall n \geq 0 .
$$

Comme $\left\{z_{n}\right\}$ et $\left\{g\left(u_{n}\right)\right\}$ convergent vers $z^{*}, g\left(u^{*}\right)$ respectivement et puisque toutes les hypothèses de la proposition 1.11 sont satisfaites, alors on peut passer à la limite dans la dernière relation et on obtient :

$$
z^{*}-g\left(u^{*}\right) \in N^{P}\left(C\left(u^{*}\right), g\left(u^{*}\right)\right)
$$

d'où

et donc

$$
\left\{h\left(y^{*}, g\left(w^{*}\right)\right)-\rho\left[T\left(x^{*}\right)-f\right]\right\} \in N^{P}\left(C\left(u^{*}\right), g\left(u^{*}\right)\right)
$$

$$
\left\{h\left(G\left(u^{*}\right), g\left(S\left(u^{*}\right)\right)\right)-\rho\left[T\left(F\left(u^{*}\right)\right)-f\right]\right\} \bigcap N^{P}\left(C\left(u^{*}\right), g\left(u^{*}\right)\right) \neq \emptyset
$$

c.-à-d. $u^{*}$ est une solution du (PQVIN). Ceci termine la preuve.

\subsection{F n'est pas nécessairement fortement monotone relativement à $T$}

Nous supposons que $F=F_{1}+F_{2}$, avec $F_{1}$ est lipschitzienne au sens de Hausdorff, fortement monotone relativement à $T$ dans $H$, et $F_{2}$ est seulement lipschitzienne au sens de Hausdorff dans $H$. Nous proposons l'algorithme suivant pour résoudre le problème (PQVIN) associé à $F=F_{1}+F_{2}$ :

\section{Algorithme 3.4.}

Étape 1. Fixer $u_{0} \in H$ et $\rho>0$ tels que :

$$
g\left(u_{0}\right) \in C\left(u_{0}\right), x_{0}^{1} \in F_{1}\left(u_{0}\right), x_{0}^{2} \in F_{2}\left(u_{0}\right), y_{0} \in G\left(u_{0}\right), w_{0} \in S\left(u_{0}\right) .
$$

Étape 2. Pour $n \geq 0$ :

Calculer : $l_{n}=h\left(y_{n}, g\left(w_{n}\right)\right)-\rho\left[T\left(x_{n}^{1}\right)+T\left(x_{n}^{2}\right)-f\right]$ et $z_{n+1}=g\left(u_{n}\right)+l_{n}$.

Choisir : $u_{n+1} \in H$ tel que $g\left(u_{n+1}\right) \in \operatorname{Proj}_{C\left(u_{n}\right)}\left(z_{n+1}\right)$.

Choisir : $x_{n+1}^{1} \in F_{1}\left(u_{n+1}\right), x_{n+1}^{2} \in F_{2}\left(u_{n+1}\right), y_{n+1} \in G\left(u_{n+1}\right), w_{n+1} \in S\left(u_{n+1}\right)$. 


\section{Hypothèses (H4)}

(1) Les constantes $\alpha_{1}, \beta, \mu, \eta, \sigma, k, k_{1}, k_{2}, \gamma_{1}, \gamma_{2}, \zeta$ et $\theta$ vérifient les inégalités suivantes :

- $\gamma_{1}>\gamma_{2}$

- $\alpha_{1}^{2} \zeta>\mu^{2} \zeta\left(\gamma_{1}^{2}-\gamma_{2}^{2}\right)+2 \mu \alpha_{1} \gamma_{2}\left(1-R-k-\sigma \zeta-R_{1} \zeta\right)$.

Théorème 3.5. Supposons que les hypothèses (2)-(6) dans (H1), les hypothèses (1)-(2) dans (H2), les hypothèses (1)-(3) dans (H3) et toutes les hypothèses (H4) sont satisfaites et supposons que le paramètre $\rho$ vérifie les inégalités :

$$
\begin{gathered}
\rho>\frac{\alpha_{1} \zeta-\mu \gamma_{2}\left(1-R-k-\sigma \zeta-R_{1} \zeta\right)}{\mu^{2} \zeta\left(\gamma_{1}^{2}-\gamma_{2}^{2}\right)}-\epsilon \\
\rho<\min \left\{\frac{\alpha_{1} \zeta-\mu \gamma_{2}\left(1-R-k-\sigma \zeta-R_{1} \zeta\right)}{\mu^{2} \zeta\left(\gamma_{1}^{2}-\gamma_{2}^{2}\right)}+\epsilon, \frac{\left(1-R-k-\sigma \zeta-R_{1} \zeta\right)}{\mu \gamma_{2} \zeta}, \frac{a-\left\|h\left(y_{n}, g\left(u_{n}\right)\right)\right\|}{\left\|T\left(x_{n}^{1}\right)+T\left(x_{n}^{2}\right)-f\right\|}\right\}
\end{gathered}
$$

où

$$
\epsilon=\frac{\sqrt{\alpha_{1}^{2}-\mu^{2}\left(\gamma_{1}^{2}-\gamma_{2}^{2}\right)+\mu^{2} \gamma_{1}^{2}\left(\frac{1-R-k}{\zeta}-R_{1}-\sigma\right)^{2}-2 \alpha_{1} \gamma_{2}\left(\frac{1-R-k}{\zeta}-R_{1}-\sigma\right)}}{\zeta \mu^{2}\left(\gamma_{1}^{2}-\gamma_{2}^{2}\right)} .
$$

Alors les suites $\left\{u_{n}\right\},\left\{x_{n}^{1}\right\},\left\{x_{n}^{2}\right\},\left\{y_{n}\right\},\left\{w_{n}\right\}$, et $\left\{z_{n}\right\}$ engendrées par l'algorithme 3.4 sont fortement convergentes vers des éléments $u^{*}, x^{1}, x^{2}, y^{*}, w^{*}$ et $z^{*}$ dans $H$ respectivement, avec $u^{*}$ une solution de (PQVIN) associée à $F=F_{1}+F_{2}$.

Preuve. En adaptant la preuve du théorème 3.2 d'une façon similaire à celle de la preuve du théorème 2.10, on peut prouver le théorème 3.5 .

\section{Sensibilité des (PQVIN)}

Notre objectif dans cette section est d'étudier la sensibilité des problèmes variationnels du type (PQVIN). Pour ceci nous considérons la famille $(\mathrm{PQVIN})_{\epsilon}$ des problèmes perturbés suivants :

trouver $u^{*} \in H$ et $\rho>0$ tels que

$$
0 \in h\left(G\left(u^{*}, \epsilon\right), g\left(S\left(u^{*}, \epsilon\right), \epsilon\right), \epsilon\right)-\rho\left[T\left(F\left(u^{*}, \epsilon\right), \epsilon\right)-f\right]-N\left(C\left(u^{*}, \epsilon\right), g\left(u^{*}, \epsilon\right)\right) . \quad(\mathrm{PQVIN})_{\epsilon}
$$

Pour mettre au clair nos idées nous considérons le cas particulier suivant de (PQVIN) ${ }_{\epsilon}(G=F=S=i d$ et $f=0)$ :

$$
\text { trouver } u^{*} \in H \text { et } \rho>0 \text { tels que } 0 \in h\left(u^{*}, g\left(u^{*}\right), \epsilon\right)-\rho T\left(u^{*}, \epsilon\right)-N\left(C\left(u^{*}, \epsilon\right), g\left(u^{*}\right)\right) \text {. }
$$

Soit $M$ un ouvert de $H$ dans lequel le paramètre $\epsilon$ varie. Nous allons utiliser les notations suivantes $T_{\epsilon}(u):=$ $T(u, \epsilon), h_{\epsilon}(u, v):=h(u, v, \epsilon), C_{\epsilon}(u):=C(u, \epsilon)$. Pour étudier la sensibilité de (PQVIN $)_{\epsilon}$, nous supposons l'existence d'une solution unique $\bar{u}$ de (PQVIN) $\bar{\epsilon}$, pour un paramètre $\bar{\epsilon} \in M$. Ensuite, nous cherchons les conditions et les hypothèses pour lesquelles, nous avons pour tout $\epsilon$ proche de $\bar{\epsilon}$, le problème (PQVIN) $)_{\epsilon}$ admet une solution unique $u(\epsilon)$ proche de $\bar{u}$. Aussi nous étudions la continuité de la fonction $\epsilon \mapsto u(\epsilon)$ autour du paramètre $\bar{\epsilon}$.

Pour cette étude nous aurons besoin des hypothèses suivantes :

\section{Hypothèses (H5) :}

(1) $g$ est $\alpha$-fortement monotone et $\sigma$-lipschitzienne.

(2) $(u, v) \mapsto h_{\epsilon}(u, v)$ est $k_{1}$-lipschitzienne par rapport à la première variable et $k_{2}$-lipschitzienne par rapport à la deuxième variable.

(3) $T_{\epsilon}$ est $\delta$-lipschitzienne, pour tout $\epsilon \in M$. 
(4) $(x, \epsilon) \mapsto C_{\epsilon}(x)$ est $\left(\kappa_{1}, \kappa_{2}\right)$-lipschitzienne avec $0<\kappa_{1}<1$ et $\kappa_{2}>0$, c.-à-d.,

$$
\left|d\left(C_{\epsilon}(x), y\right)-d\left(C_{\epsilon}\left(x^{\prime}\right), y^{\prime}\right)\right| \leq\left\|y-y^{\prime}\right\|+\kappa_{1}\left\|x-x^{\prime}\right\|+\kappa_{2}\|\epsilon-\alpha\|
$$

pour tout $x, x^{\prime}, y, y^{\prime} \in H$ et pour tout $\epsilon, \alpha \in M$. Nous supposons aussi que pour tout $x \in H$ et pour tout $\epsilon \in M$, les images $C_{\epsilon}(x)$ sont uniformément $r^{\prime}$-prox-régulier, avec $r^{\prime}>0$ et $\left.r \in\right] 0, r^{\prime}$ [ et nous notons $\operatorname{par} \zeta:=\frac{r^{\prime}}{r^{\prime}-r}$.

(5) La projection vérifie l'inégalité suivante avec $0<k<1$ et $k^{\prime}>0$

$$
\left\|\operatorname{Proj}_{C_{\epsilon}(x)}(z)-\operatorname{Proj}_{C_{\alpha}(y)}(z)\right\| \leq k\|x-y\|+k^{\prime}\|\epsilon-\alpha\|,
$$

pour tout $z, x, y \in H$ et pour tout $\epsilon, \alpha \in M$.

(6) Les applications $\epsilon \mapsto h_{\epsilon}(\cdot, \cdot)$ et $\epsilon \mapsto T_{\epsilon}(\cdot)$ sont $\beta_{1}$-lipschitzienne et $\beta_{2}$-lipschitzienne respectivement.

(7) Les constantes $\alpha, \sigma, k, k_{1}, k_{2}$ et $\zeta$ vérifient : $\left.R+k+\zeta \sqrt{\sigma^{2}+\left(k_{1}+k_{2} \sigma\right)^{2}}\right)<1$.

Le lemme suivant est l'un des points clés de notre résultat principal de cette section.

Lemme 4.1. Supposons que $u$ est un point fixe de l'application $u \mapsto L(u, \epsilon)$, pour tout $\epsilon \in M$, où

$$
L(u, \epsilon)=u-g(u)+\operatorname{Proj}_{C_{\epsilon}(u)}\left(h_{\epsilon}(u, g(u))-\rho T_{\epsilon}(u)+g(u)\right) .
$$

Alors $u$ est une solution de $(P Q V I N)_{\epsilon}$.

Preuve. Soit $u$ un point fixe de l'application $u \mapsto L(u, \epsilon)$, pour tout $\epsilon \in M$, c.-à-d. pour tout $\epsilon \in M$ on a

$$
u=L(u, \epsilon)=u-g(u)+\operatorname{Proj}_{C_{\epsilon}(u)}\left(h_{\epsilon}(u, g(u))-\rho T_{\epsilon}(u)+g(u)\right)
$$

D'où

$$
g(u)=\operatorname{Proj}_{C_{\epsilon}(u)}\left(h_{\epsilon}(u, g(u))-\rho T_{\epsilon}(u)+g(u)\right)
$$

et donc d'après la définition du cône normal proximal on obtient

$$
h_{\epsilon}(u, g(u))-\rho T_{\epsilon}(u) \in N^{P}\left(C_{\epsilon}(u) ; g(u)\right),
$$

ce qui assure que $u$ est une solution de $(\mathrm{PQVIN})_{\epsilon}$.

Lemme 4.2. Soient $\bar{\epsilon} \in M$ et $\bar{u}$ une solution de $(P Q V I N)_{\bar{\epsilon}}$. Soient $b_{1}, b_{2}>0$ et $V_{1}=\bar{u}+b_{1} \mathbb{B}$ et $V_{2}=$ $\bar{\epsilon}+b_{2} \mathbb{B}$. Supposons que les hypothèse (H5) sont satisfaites et que pour tout $\epsilon \in V_{2}$, les application $u \mapsto$ $h_{\epsilon}(u, g(u))$ et $u \mapsto T_{\epsilon}(u)$ sont bornées par $A_{1}>0$ et $A_{2}>0$ respectivement. Supposons aussi que les constantes $b_{1}, b_{2}, A_{1}, A_{2}, \sigma, \kappa_{1}, \kappa_{2}$ et le paramètre $\rho>0$ vérifient l'inégalité suivante:

$$
A_{1}+\rho A_{2}+(\sigma+1) \kappa_{1} b_{1}+\kappa_{2} b_{2}<r \text {. }
$$

Alors pour tout $u \in V_{1}$ et tout $\epsilon \in V_{2}$, on a

$$
\left.h_{\epsilon}(u, g(u))-\rho T_{\epsilon}(u)+g(u) \in\left[C_{\epsilon}(u)\right]_{r}, \quad \text { (l'élargissement de } C_{\epsilon}(u)\right) \text {. }
$$


Preuve. Soient $u \in V_{1}$ et $\epsilon \in V_{2}$. Puisque $\bar{u}$ est une solution de (PQVIN) $)_{\bar{\epsilon}}$ on a $g(\bar{u}) \in C_{\bar{\epsilon}}(\bar{u})$ et donc

$$
\begin{aligned}
d\left(h_{\epsilon}(u, g(u))-\rho T_{\epsilon}(u)+g(u) ; C_{\epsilon}(u)\right)= & d\left(h_{\epsilon}(u, g(u))-\rho T_{\epsilon}(u)+g(u) ; C_{\epsilon}(u)\right) \\
& -d\left(g(\bar{u}) ; C_{\bar{\epsilon}}(\bar{u})\right) \\
\leq & \left\|h_{\epsilon}(u, g(u))-\rho T_{\epsilon}(u)+g(u)-g(\bar{u})\right\|+ \\
& +\kappa_{1}\|u-\bar{u}\|+\kappa_{2}\|\epsilon-\bar{\epsilon}\| \\
\leq & \left\|h_{\epsilon}(u, g(u))-\rho T_{\epsilon}(u)\right\|+\sigma\|u-\bar{u}\|+ \\
& \kappa_{1} b 1+\kappa_{2} b 2 \\
\leq & A_{1}+\rho A_{2}+(\sigma+1) \kappa_{1} b_{1}+\kappa_{2} b_{2}<r .
\end{aligned}
$$

Donc $h_{\epsilon}(u, g(u))-\rho T_{\epsilon}(u)+g(u) \in\left[C_{\epsilon}(u)\right]_{r}$, ce qui termine la preuve.

Lemme 4.3. Sous les mêmes hypothèses du lemme 4.2 on a pour tout $u_{1}$ et $u_{2}$ dans $V_{1}$ et tout $\epsilon_{1}$ et $\epsilon_{2} \in V_{2}$ :

$$
\left\|L\left(u_{1}, \epsilon_{1}\right)-L\left(u_{2}, \epsilon_{2}\right)\right\| \leq \gamma_{1}\left\|u_{1}-u_{2}\right\|+\gamma_{2}\left\|\epsilon_{1}-\epsilon_{2}\right\|,
$$

où

$$
\gamma_{1}=\left(\sqrt{1-2 \alpha+\sigma^{2}}+k+\zeta\left[\sqrt{\sigma^{2}+\left(k_{1}+k_{2} \sigma\right)^{2}}+\rho \delta\right]\right) \text { et } \gamma_{2}=k^{\prime}+\zeta\left[\beta_{1}+\rho \beta_{2}\right] .
$$

Preuve. D'après la définition de l'application $u \mapsto L(u, \epsilon)$ on a pour tout $\epsilon \in V_{2}$

$$
\begin{aligned}
& L\left(u_{1}, \epsilon\right)-L\left(u_{2}, \epsilon\right)=u_{1}-u_{2}-g\left(u_{1}\right)+g\left(u_{2}\right)-\operatorname{Proj}_{C_{\epsilon}\left(u_{2}\right)}\left(h_{\epsilon}\left(u_{2}, g\left(u_{2}\right)\right)+\rho T_{\epsilon}\left(u_{2}\right)\right. \\
& \left.+g\left(u_{2}\right)\right)+\operatorname{Proj}_{C_{\epsilon}\left(u_{1}\right)}\left(h_{\epsilon}\left(u_{2}, g\left(u_{2}\right)\right)-\rho T_{\epsilon}\left(u_{2}\right)+g\left(u_{2}\right)\right)-\operatorname{Proj}_{C_{\epsilon}\left(u_{1}\right)}\left(h_{\epsilon}\left(u_{2}, g\left(u_{2}\right)\right)\right. \\
& \left.-T_{\epsilon}\left(u_{2}\right)+g\left(u_{2}\right)\right)+\operatorname{Proj}_{C_{\epsilon}\left(u_{1}\right)}\left(h_{\epsilon}\left(u_{1}, g\left(u_{1}\right)\right)-\rho T_{\epsilon}\left(u_{1}\right)+g\left(u_{1}\right)\right) .
\end{aligned}
$$

En utilisant l'hypothèse (7) dans (H5), le lemme 4.2 et la proposition 4.3-(3), on obtient

$$
\begin{aligned}
\left\|L\left(u_{1}, \epsilon\right)-L\left(u_{2}, \epsilon\right)\right\| \leq & \left\|u_{1}-u_{2}-g\left(u_{1}\right)+g\left(u_{2}\right)\right\|+k\left\|u_{1}-u_{2}\right\|+\zeta \| h_{\epsilon}\left(u_{2}, g\left(u_{2}\right)\right) \\
& -h_{\epsilon}\left(u_{1}, g\left(u_{1}\right)\right)+g\left(u_{2}\right)-g\left(u_{1}\right)\|+\zeta \rho\| T_{\epsilon}\left(u_{2}\right)-T_{\epsilon}\left(u_{1}\right) \| .
\end{aligned}
$$

Puisque $g$ est fortement monotone avec une constante $\alpha$ et d'après la propriété de Lipschitz de $g$ on a

$$
\begin{aligned}
\left\|u_{1}-u_{2}-g\left(u_{1}\right)+g\left(u_{2}\right)\right\|^{2} & \leq\left(\sigma^{2}+1\right)\left\|u_{1}-u_{2}\right\|^{2}-2<g\left(u_{1}\right)-g\left(u_{2}\right), u_{1}-u_{2}> \\
& \leq\left(1-2 \alpha+\sigma^{2}\right)\left\|u_{1}-u_{2}\right\|^{2} .
\end{aligned}
$$

D'une part, la propriété de Lipschitz de $g$ et de $h$ nous assure

$$
\left\|h_{\epsilon}\left(u_{2}, g\left(u_{2}\right)\right)-h_{\epsilon}\left(u_{1}, g\left(u_{1}\right)\right)+g\left(u_{2}\right)-g\left(u_{1}\right)\right\|^{2} \leq\left[\sigma^{2}+\left(k_{2} \sigma+k_{1}\right)^{2}\right]\left\|u_{1}-u_{2}\right\|^{2} .
$$

Finalement, en combinant les inégalités (4.1), (4.2) et (4.3) et en utilisant la propriété $\delta$-Lipschitz de $T_{\epsilon}$, on obtient

$$
\left\|L\left(u_{1}, \epsilon\right)-L\left(u_{2}, \epsilon\right)\right\|=\left(\sqrt{1-2 \alpha+\sigma^{2}}+k+\zeta\left[\sqrt{\sigma^{2}+\left(k_{1}+k_{2} \sigma\right)^{2}}+\rho \delta\right]\right)\left\|u_{1}-u_{2}\right\| .
$$


D'autre part on a pour tout $u$ dans $V_{1}$ et tout $\epsilon_{1}$ et $\epsilon_{2} \in V_{2}$

$$
\begin{aligned}
\left\|L\left(u, \epsilon_{1}\right)-L\left(u, \epsilon_{2}\right)\right\|= & \| \operatorname{Proj}_{C_{\epsilon_{1}}(u)}\left(h_{\epsilon_{1}}(u, g(u))-\rho T_{\epsilon_{1}}(u)+g(u)\right) \\
& -\operatorname{Proj}_{C_{\epsilon_{2}}(u)}\left(h_{\epsilon_{2}}(u, g(u))-\rho T_{\epsilon_{2}}(u)+g(u)\right) \| \\
\leq & \| \operatorname{Proj}_{\epsilon_{\epsilon_{1}}(u)}\left(h_{\epsilon_{1}}(u, g(u))-\rho T_{\epsilon_{1}}(u)+g(u)\right) \\
& -\operatorname{Proj}_{C_{\epsilon_{2}}(u)}\left(h_{\epsilon_{1}}(u, g(u))-\rho T_{\epsilon_{1}}(u)+g(u)\right) \| \\
& +\| \operatorname{Proj}_{C_{\epsilon_{2}}(u)}\left(h_{\epsilon_{1}}(u, g(u))-\rho T_{\epsilon_{1}}(u)+g(u)\right) \\
& -\operatorname{Proj}_{C_{\epsilon_{2}}(u)}\left(h_{\epsilon_{2}}(u, g(u))-\rho T_{\epsilon_{2}}(u)+g(u)\right) \| \\
\leq & k^{\prime}\left\|\epsilon_{1}-\epsilon_{2}\right\|+\zeta\left\|\left[h_{\epsilon_{2}}(u, g(u))-\rho T_{\epsilon_{2}}(u)\right]-\left[h_{\epsilon_{1}}(u, g(u))-\rho T_{\epsilon_{1}}(u)\right]\right\| \\
\leq & k^{\prime}\left\|\epsilon_{1}-\epsilon_{2}\right\|+\zeta\left\|h_{\epsilon_{2}}(u, g(u))-h_{\epsilon_{1}}(u, g(u))\right\|+\rho \zeta\left\|T_{\epsilon_{2}}(u)-T_{\epsilon_{1}}(u)\right\| \\
\leq & k^{\prime}\left\|\epsilon_{1}-\epsilon_{2}\right\|+\zeta \beta_{1}\left\|\epsilon_{1}-\epsilon_{2}\right\|+\rho \zeta \beta_{2}\left\|\epsilon_{1}-\epsilon_{2}\right\| \\
= & {\left[k^{\prime}+\zeta \beta_{1}+\rho \zeta \beta_{2}\right]\left\|\epsilon_{1}-\epsilon_{2}\right\|=\gamma_{2}\left\|\epsilon_{1}-\epsilon_{2}\right\| . }
\end{aligned}
$$

Soit maintenant $u_{1}$ et $u_{2}$ dans $V_{1}$ et $\epsilon_{1}$ et $\epsilon_{2} \in V_{2}$. Alors on a

$$
\begin{aligned}
\left\|L\left(u_{1}, \epsilon_{1}\right)-L\left(u_{2}, \epsilon_{2}\right)\right\| & \leq\left\|L\left(u_{1}, \epsilon_{1}\right)-L\left(u_{2}, \epsilon_{1}\right)\right\|+\left\|L\left(u_{2}, \epsilon_{1}\right)-L\left(u_{2}, \epsilon_{2}\right)\right\| \\
& \leq \gamma_{1}\left\|u_{1}-u_{2}\right\|+\gamma_{2}\left\|\epsilon_{1}-\epsilon_{2}\right\| .
\end{aligned}
$$

Ceci termine la preuve de ce lemme.

Remarque 4.4. Si on suppose que le paramètre $\rho$ vérifie l'inégalité suivante :

$$
\rho<\frac{1-k-\sqrt{1-2 \alpha+\sigma^{2}}-\zeta \sqrt{\sigma^{2}+\left(k_{1}+k_{2} \sigma\right)^{2}}}{\zeta \delta}
$$

on a la constante $\gamma_{1}$ est toujours strictement inférieure à 1 et on obtient donc comme conséquence directe du lemme 4.3, que l'application $u \mapsto L(u, \epsilon)$ est lipschitzienne sur $V_{1}$ avec une constante $\gamma_{1}<1$ et donc elle admet admet un point fixe $u(\epsilon)$ dans $V_{1}$ pour tout $\epsilon \in V_{2}$, c.-à-d.,

$$
u(\epsilon)=L(u(\epsilon), \epsilon)
$$

Ceci assure d'après le lemme 4.1 que ce point fixe $u(\epsilon)$ est une solution du problème perturbé $(\mathrm{PQVIN})_{\epsilon}$. En particulier, pour $\epsilon=\bar{\epsilon}$ on a

$$
u(\bar{\epsilon})=\bar{u}=L(u(\bar{\epsilon}), \bar{\epsilon}),
$$

qui est une solution du problème perturbé (PQVIN) $)_{\bar{\epsilon}}$.

En utilisant le lemme 4.3 nous allons prouver dans le lemme suivant la propriété de Lipschitz de la fonction $\epsilon \mapsto u(\epsilon)$.

Lemme 4.5. Supposons que les même hypothèses dans le lemme 4.3 sont satisfaites. Alors la fonction $\epsilon \mapsto u(\epsilon)$ est $\beta$-lipschitzienne sur $V_{2}$ avec $\beta=\frac{\gamma_{2}}{1-\gamma_{1}}$.

Preuve. Soient $\epsilon_{1}$ et $\epsilon_{2} \in V_{2}$. Alors

$$
\begin{aligned}
\left\|u\left(\epsilon_{1}\right)-u\left(\epsilon_{2}\right)\right\| & =\left\|L\left(u\left(\epsilon_{1}\right), \epsilon_{1}\right)-L\left(u\left(\epsilon_{2}\right), \epsilon_{2}\right)\right\| \\
& \leq \gamma_{1}\left\|u\left(\epsilon_{1}\right)-u\left(\epsilon_{2}\right)\right\|+\gamma_{2}\left\|\epsilon_{1}-\epsilon_{2}\right\| .
\end{aligned}
$$

D'où

$$
\left(1-\gamma_{1}\right)\left\|u\left(\epsilon_{1}\right)-u\left(\epsilon_{2}\right)\right\| \leq \gamma_{2}\left\|\epsilon_{1}-\epsilon_{2}\right\|
$$


Puisque $\left.\gamma_{1} \in\right] 0,1[$ alors on obtient

$$
\left\|u\left(\epsilon_{1}\right)-u\left(\epsilon_{2}\right)\right\| \leq \frac{\gamma_{2}}{1-\gamma_{1}}\|\epsilon-\bar{\epsilon}\|
$$

ce qui termine la preuve.

Le théorème suivant est notre résultat principal de cette section. Sa preuve est une simple combinaison des lemmes précédents.

Théorème 4.6. Soit $\bar{u}$ une solution unique de $(P Q V I N)_{\bar{\epsilon}}$, pour un paramètre $\bar{\epsilon} \in M$. Supposons que les hypothèses (H5) soient satisfaites. Supposons aussi que le paramètre $\rho$ vérifie (4.4). Alors il existe un voisinage $V$ dans $M$ de $\bar{\epsilon}$ tel que pour tout $\epsilon \in V$ il existe une unique solution $u(\epsilon)$ de $(P Q V I N)_{\epsilon}$ proche de $\bar{u}$ et la fonction $\epsilon \mapsto u(\epsilon)$ est lipschitzienne autour de $\bar{\epsilon}$ dans $V$.

Remerciements. Les auteurs remercient les deux rapporteurs pour la lecture prudente et minutieuse du papier. Leurs suggestions et leurs remarques pertinentes ont permis de nombreuses améliorations dans le papier.

\section{RÉFÉRENCES}

[1] M. Bounkhel, L. Tadj and A. Hamdi, Iterative Schemes to Solve Non convex Variational Problems. J. Ineq. Pure Appl. Math. 4 (2003), Article 14.

[2] M. Bounkhel and L. Thibault, On various notions of regularity of sets in non smooth analysis. Nonlinear Anal. Theory Methods Appl. 48 (2002) 223-246.

[3] M. Bounkhel and L. Thibault, Further characterizations of regular sets in Hilbert spaces and their applications to nonconvex sweeping process. Preprint, Centro de Modelamiento Matematico (CMM), Universidad de Chile (2000). Submitted to J. Nonlinear Convex Anal.

[4] C. Castaing and M. Valadier, Convex Analysis and Measurable Multifunctions. Lect. Notes Math. 580 (1977).

[5] Y.J. Cho, Z. He, Y.F. Cao and N.J. Huang, On the generalized strongly nonlinear implicit quasivariational inequalities for set-valued mappings. J. Ineq. Pure Appl. Math. 1 (2000), Article 15.

[6] F.H. Clarke, Optimization and Nonsmooth Analysis. Wiley-Interscience, New York (1983).

[7] F.H. Clarke, R.J. Stern and P.R. Wolenski, Proximal smoothness and the lower $C^{2}$-property. J. Convex Anal. 2 (1995) 117-144.

[8] F.H. Clarke, Yu.S. Ledyaev, R.J. Stern and P.R. Wolenski, Nonsmooth Analysis and Control Theory. Springer-Verlag, New York (1998).

[9] M.A. Noor, General algorithm for variational inequalities. J. Optim. Theory Appl. 73 (1992) 409-413.

[10] P.D. Panagiotopoulos and G.E. Stavroulakis, New types of variational principles based on the notion of quasidifferentiability. Acta Mech. 94 (1992) 171-194.

[11] R.A. Poliquin, R.T. Rockafellar and L. Thibault, Local differentiability of distance functions. Trans. Amer. Math. Soc. 352 (2000) 5231-5249.

[12] R.T. Rockafellar and R. Wets, Variational Analysis. Springer-Verlag, Berlin (1998).

[13] G. Stampacchia, Formes bilinéaires coercives sur les ensembles convexes. C. R. Acad. Sci. Paris 258 (1964) $4413-4416$.

[14] L.C. Zeng, On a general projection algorithm for variational inequalities. J. Optim. Theory Appl. 97 (1998) $229-235$. 